\title{
POSITIVITY OF ZETA DISTRIBUTIONS AND SMALL UNITARY REPRESENTATIONS
}

\author{
L. BARCHINI, M. SEPANSKI, AND R. ZIERAU
}

\begin{abstract}
This paper studies the positivity of certain zeta distributions associated to simple noneuclidean Jordan algebras. The distributions are calculated in the cases where they are positive. The main technique revolves around an explicit form of the corresponding functional equation. Using an identity relating these zeta distributions to the standard intertwining operators for the associated conformal groups, explicit families of singular unitary representations are then constructed.
\end{abstract}

\section{INTRODUCTION}

The purpose of this article is to give a construction of families of singular unitary representations of certain simple Lie groups. We consider Lie groups which are related to conformal groups of noneuclidean Jordan algebras. See Table 1 for a precise list of the groups under consideration. A key property of such groups is that each has a parabolic subgroup $P=L N$ for which the adjoint action of $L$ on $\mathfrak{n}=\operatorname{Lie}(N)$ has a finite number of orbits. In particular, there is an open orbit which is in fact dense and has complement defined by a polynomial equation $P(X)=\nabla^{2}(X)=0$. It is well-known that $\nabla^{s}$ defines a family of tempered distributions (meromorphic in $s \in \mathbf{C}$ ) known as zeta distributions. This family may be regularized to give a family of distributions which is complex analytic in $s$. It turns out that this family of distributions is intimately related to intertwining operators between certain degenerate principal series representations (for $P=L N)$ and to the unitarity of certain subrepresentations. We show how these distributions play a role in giving unitary realizations of these representations.

To be slightly more precise, let $R_{s}$ be the regularized distribution corresponding to $\nabla^{-s}$; see (6.1). Then our first main theorem is that $R_{s}$ is a positive distribution if and only if $s \in(-\infty, e+1) \cup\left\{\frac{m}{n}-q d: q=\right.$ $0,1, \ldots, n-1\}$, where $d, e, m$ and $n$ are certain integers which depend on $G$. Theorem 5.12 states that for the discrete points $s=\frac{m}{n}-q d, q=0,1, \ldots, n-1, R_{s}$ is a quasi-invariant measure on an $L$-orbit $\mathcal{O}_{q}$ in $\mathfrak{n}$. Under our conditions on $G$ there are $n+1 L$-orbits $\mathcal{O}_{q}$ which we may write as $\{0\}=\mathcal{O}_{0} \subset \mathcal{O}_{1} \subset \cdots \subset \mathcal{O}_{n}$; thus each orbit corresponds to a distribution. Next we consider certain smooth degenerate principal series representations, which we denote by $I(s)$. We will use the realization of $I(s)$ as certain smooth functions on $\overline{\mathfrak{n}}$ in the usual way. By general principles there is a complex analytic family of $G$-intertwining operators

$$
A_{s}: I(s) \rightarrow I(-s)
$$

We show that for Schwartz functions $f$ on $\overline{\mathfrak{n}}$

$$
\left(A_{s} f\right)(Y)=R_{s}\left(\left(\tau_{Y} f\right)^{\uparrow}\right)
$$

2000 Mathematics Subject Classification. 22E45, 22E30.

Authors partially supported by the NSF grants: DMS 98-01605 (L.B. and R.Z.) and DMS 0074991 (L.B.) NSA grant MDA 904-02-1-0081 (M.S.). Much of this work was carried out while the authors were members at the MSRI during the fall of 2001. The second author thanks Baylor University for its Summer Sabbatical support and support while at MSRI. 
where $\tau_{Y}$ is translation by $Y$ in $\overline{\mathfrak{n}}$ and ${ }^{\widehat{ }}$ denotes Fourier transform. When $s \in \mathbf{R}$ the intertwining operator defines a $G$-invariant hermitian form (often indefinite) on the image of $A_{s}$ by

$$
\left\langle A_{s} f_{1}, A_{s} f_{2}\right\rangle=\int_{\overline{\mathfrak{n}}} f_{1}(Y) \overline{A_{s} f_{2}(Y)} d Y
$$

for functions $f_{1}$ and $f_{2}$ in $I(s)$. For the following results we exclude $G=S O(p, q)$ (in case 4 . on Table 1) as the results about unitarity are somewhat different in this case. Assume for the moment that $s=\frac{m}{n}-q d$, $q=0,1, \ldots, n-1$, so that $R_{s}$ is a quasi-invariant measure on $\mathcal{O}_{q}$. In view of (1.1), formula (1.2) for the hermitian form becomes

$$
\left\langle A_{s} f_{1}, A_{s} f_{2}\right\rangle=\int_{\mathcal{O}_{q}} \widehat{f_{1}} \overline{\hat{f}_{2}} d \nu_{q}
$$

for Schwartz functions $f_{1}$ and $f_{2}$ on $\overline{\mathfrak{n}}$. Lemma 8.10, Theorem 8.11 and Corollary 8.12 state that the completion $\mathcal{H}_{s}$ of $\operatorname{Im}\left(A_{s}\right)$ with respect to this inner product is an irreducible unitary $G$-representation. For this we show that the Fourier transform provides a factoring of $A_{s}$ through $L^{2}\left(\mathcal{O}_{q}\right)$. We show that this gives a unitary equivalence between $\mathcal{H}_{s}$ and $L^{2}\left(\mathcal{O}_{q}\right)$ as $P$-representations. Therefore the natural (irreducible) action of $P$, via the Fourier transform, on $L^{2}\left(\mathcal{O}_{q}\right)$ extends to an irreducible unitary representation of $G$. A similar result holds for the continuous parameter $s \in[0, e+1)$.

Our main technique for studying the zeta distributions is a functional equation. Functional equations in this type of setting have a long history. See for example [25], [22], [3] and [15]. The version we use is contained in [15]. The distributions $R_{s}$ are related to classical Riesz distributions and generalizations. For instance, when $G$ is the conformal group of a euclidean Jordan algebra (i.e., $G$ is of tube type) then families of Riesz distributions are associated to the open convex $L$-orbits in $\mathfrak{n}$. Many of the results of this article, such as positivity of the distributions for certain parameters and the unitary realization, hold in this setting. See for example [7], [9] and [19]. We remark that the main tool used in the case of convex $L$-orbits is the Laplace transform. In the nonconvex setting of this article the functional equation becomes the main tool.

Our method is, to some degree, inspired by the treatment of $S L(2, \mathbf{R})$ in [8] and the treatment of holomorphic representations in [19]. The representations considered in this article have been studied previously. Our Corollary 8.12 is obtained in [6] by different methods. The unitarizability of the representations is contained in [20]. There is some overlap with techniques in [1] and the recent article [12]. We thank I. Muller for several conversations and for making part of her manuscript [17] available to us.

\section{Preliminaries}

Each simple noneuclidean Jordan algebra occurs as the abelian nilradical of a maximal parabolic subalgebra of a reductive Lie algebra $\mathfrak{g}$. There is a reductive Lie group $G$, with Lie algebra $\mathfrak{g}$, having the following properties.

2.1. G contains a parabolic subgroup $P=L N$ (a Levi decomposition) such that

(1) $P$ and its opposite parabolic are $G$-conjugate, and

(2) $N$ is abelian.

(3) The symmetric space corresponding to $G$ is not of tube type.

For a given simple noneuclidean Jordan algebra the $\mathfrak{g}$ and $G$ as above are not quite unique. The choices for the groups $G$ which we work with are given in Table 1 in Appendix A. 
Therefore we make the assumption that $G$ is a group listed on Table 1. Then $G$ has a Cartan involution $\theta$ so that $\theta$ sends $P$ to the opposite parabolic. We let $K$ be the fixed point group of $\theta$, a maximal compact subgroup of $G$. As is customary we write the Lie algebra of $G$ (resp. $K$ ) as $\mathfrak{g}$ (resp. $\mathfrak{k}$ ). The Cartan involution determines a Cartan decomposition $\mathfrak{g}=\mathfrak{k}+\mathfrak{s}$.

Following [13] there is an abelian subalgebra $\mathfrak{b}$ of $\mathfrak{l} \cap \mathfrak{s}$ with the following properties.

(1) There are commuting copies of $\mathfrak{s l}(2, \mathbf{R})$ in $\mathfrak{g}$ spanned by $\left\{F_{j}, H_{j}, E_{j}\right\}$, a standard basis in the sense that

$$
\begin{aligned}
& \theta\left(E_{j}\right)=-F_{j} \text { and } \theta\left(H_{j}\right)=-H_{j}, \\
& {\left[E_{j}, F_{j}\right]=H_{j},\left[H_{j}, E_{j}\right]=2 E_{j} \text { and }\left[H_{j}, F_{j}\right]=-2 F_{j}}
\end{aligned}
$$

with $E_{j} \in \mathfrak{n}, F_{j} \in \overline{\mathfrak{n}}$ and $\mathfrak{b}=\sum_{j=1}^{n} \mathbf{R} H_{j}$.

(2) For $\epsilon_{k}\left(\sum_{j=1}^{n} a_{j} H_{j}\right) \equiv a_{k}$, the $\mathfrak{b}$-roots in $\mathfrak{g}, \mathfrak{l}$ and $\mathfrak{n}$ are

$$
\begin{aligned}
& \Sigma(\mathfrak{g}, \mathfrak{b})=\left\{ \pm\left(\epsilon_{j}-\epsilon_{k}\right): 1 \leq j<k \leq n\right\} \cup\left\{ \pm\left(\epsilon_{j}+\epsilon_{k}\right): 1 \leq j, k \leq n\right\}, \\
& \Sigma(\mathfrak{l}, \mathfrak{b})=\left\{ \pm\left(\epsilon_{j}-\epsilon_{k}\right): 1 \leq j<k \leq n\right\} \text { and } \\
& \Sigma(\mathfrak{n}, \mathfrak{b})=\left\{\epsilon_{j}+\epsilon_{k}: 1 \leq j, k \leq n\right\} .
\end{aligned}
$$

For each $G$ the roots in $\mathfrak{n}$ have just two multiplicities, defining integers ${ }^{1} d$ and $e$ :

$$
\text { each short root has multiplicity } 2 d \text { and }
$$$$
\text { each long root has multiplicity } e+1 \text {. }
$$

Define

$$
\Sigma^{+}(\mathfrak{g}, \mathfrak{b})=\left\{\epsilon_{j}-\epsilon_{k}: 1 \leq j<k \leq n\right\} \cup \Sigma(\mathfrak{n}, \mathfrak{b})
$$

Definition 2.4. Taking $n=\operatorname{dim}(\mathfrak{b})$ as above, we make the following definitions.

(1) The rank of $\mathfrak{n}$ is $n$.

(2) $\Lambda_{0} \equiv \sum_{j=1}^{n} \epsilon_{j}$.

(3) $\chi_{q}$ is the positive character of $L$ with differential $2 q d \Lambda_{0}$ for $q=0,1,2, \ldots n$.

We will often denote $\chi_{1}$ by $\chi$. The following lemma is easily proved.

Lemma 2.5. Set $m \equiv \operatorname{dim}(\mathfrak{n})$ and $\rho(\mathfrak{n}) \equiv \frac{1}{2} \sum_{\alpha \in \Sigma(\mathfrak{n}, \mathfrak{b})} \alpha$. Then

(1) $m=n(d(n-1)+(e+1))$,

(2) $\rho(\mathfrak{n})=\frac{m}{n} \Lambda_{0}$,

(3) $\left|\operatorname{det}\left(\left.A d(\ell)\right|_{\mathfrak{n}}\right)\right|=\chi(\ell)^{\frac{m}{d n}}$.

The integers $n, m, d$ and $e$ are listed on Table 1 for each group.

The orbit structure of $L$ acting on $\mathfrak{n}$ will play an important role. If we set

$$
X_{q} \equiv E_{1}+\cdots+E_{q}, q=1,2, \ldots, n \text { and } X_{0} \equiv 0
$$

then by [16] and [11] the $L$-orbits in $\mathfrak{n}$ are precisely

$$
\mathcal{O}_{q}=L\left(X_{q}\right), q=0,1,2, \ldots, n .
$$

\footnotetext{
${ }^{1}$ In the case of $S O(p, q)$, Case 4 on Tables 1 and $2, d$ is a half-integer. When $n=1, d$ is zero.
} 
We write $\mathcal{O}_{q}=L / S_{q}, S_{q}$ the stabilizer of $X_{q}$. As $\operatorname{ad}\left(X_{n}\right): \mathfrak{l} \rightarrow \mathfrak{n}$ is onto, $\mathcal{O}_{n}$ is open in $\mathfrak{n}$; it is also dense. The orbit $\mathcal{O}_{n}$ is a semisimple symmetric space. Consider

$$
\tau=\operatorname{Ad}\left(\prod_{j=1}^{n} \exp \left(\frac{\pi}{2}\left(E_{j}-F_{j}\right)\right)\right)
$$

Then $\tau(\mathfrak{l}) \subset \mathfrak{l}, \tau(\mathfrak{n}) \subset \overline{\mathfrak{n}}$ and $\tau(\overline{\mathfrak{n}}) \subset \mathfrak{n}$. Furthermore, $\left.\tau\right|_{\mathfrak{l}}$ is an involution and $\mathfrak{s}_{n}$, the Lie algebra of $S_{n}$, is the subalgebra of $\mathfrak{l}$ fixed by $\tau$. Therefore, $L / S_{n}$ is a semisimple symmetric space of rank $n$. We will describe the other orbits and stabilizers in some detail in Section 3.

There is a diffeomorphism of $\overline{\mathfrak{n}} \times L \times \mathfrak{n}$ onto a dense open set in $G$ given by $(Y, \ell, X) \rightarrow \bar{n}_{Y} \ell n_{X}$, where $\bar{n}_{Y}=\exp (Y)$ and $n_{X}=\exp (X)$. Therefore, on a dense open subset of $G$, there is a decomposition $g=$ $\bar{n}_{Y} \ell n_{X}$. Furthermore, $L=M A$ where $A=\exp (\mathfrak{a}), \mathfrak{a} \equiv \bigcap_{j<k} k e r\left(\epsilon_{j}-\epsilon_{k}\right)$. In particular, the $L$ part of the decomposition has a component in $A$. We define $a(g) \in A$ by

$$
g \in \bar{N} M a(g) N
$$

By 2.1 there is a $w \in K$ so that $\operatorname{Ad}(w) \mathfrak{n}=\overline{\mathfrak{n}}$. In particular we may define functions on dense open subsets of $\overline{\mathfrak{n}}$ and $\mathfrak{n}$ by

$$
\begin{aligned}
& \bar{\nabla}(Y) \equiv \mathrm{e}^{\Lambda_{0}\left(\log \left(a\left(w \bar{n}_{Y}\right)\right)\right)}, Y \in \overline{\mathfrak{n}} \quad \text { and } \\
& \nabla(X) \equiv \bar{\nabla}(\theta(X)), X \in \mathfrak{n} .
\end{aligned}
$$

Lemma 2.10. $\bar{\nabla}(Y)$ (respectively $\nabla(X)$ ) extends to a well-defined function on $\overline{\mathfrak{n}}$ (respectively $\mathfrak{n}$ ) and the following hold.

(1) $\bar{\nabla}(Y)^{2}$ (respectively $\left.\nabla(X)^{2}\right)$ is a homogeneous polynomial on $\overline{\mathfrak{n}}$ (respectively $\mathfrak{n}$ ) of degree $2 n$.

(2) $\bar{\nabla}(\ell \cdot Y)=\left|\operatorname{det}\left(\left.A d(\ell)\right|_{\overline{\mathfrak{n}}}\right)\right|^{\frac{n}{m}} \bar{\nabla}(Y)=\chi(\ell)^{-\frac{1}{d}} \bar{\nabla}(Y)$ for $\ell \in L$ and $Y \in \overline{\mathfrak{n}}$, and $\nabla(\ell \cdot X)=\left|\operatorname{det}\left(\left.A d(\ell)\right|_{\mathfrak{n}}\right)\right|^{\frac{n}{m}} \nabla(X)=\chi(\ell)^{\frac{1}{d}} \nabla(X)$ for $\ell \in L$ and $X \in \mathfrak{n}$.

(3) Both $\bar{\nabla}$ and $\nabla$ are invariant under $L \cap K$.

Proof. Consider a Cartan subalgebra $\mathfrak{h}$ of $\mathfrak{g}$ containing $\mathfrak{b}$. Choose a positive system of $\mathfrak{h}$-roots $\Sigma^{+}(\mathfrak{g}, \mathfrak{h})$ with the property that the positive $\mathfrak{h}$-roots restrict exactly to the roots in $\Sigma^{+}(\mathfrak{g}, \mathfrak{b})$. Let $\tilde{\Lambda}_{0}$ be the extension of $\Lambda_{0}$ to $\mathfrak{h}$ (by 0 on $\mathfrak{b}^{\perp}$ ). Then $2 \tilde{\Lambda}_{0}$ is dominant and analytically integral (since each $2 \epsilon_{j}$ is a root). In particular there is a finite dimensional representation $U_{1}$ of $G$ with highest weight $2 \tilde{\Lambda}_{0}$. Fix an inner product $\langle$,$\rangle so$ that $\langle g u, v\rangle=\left\langle u, \theta\left(g^{-1}\right) v\right\rangle$, for $u, v \in U_{1}$ and $g \in G$. Then for a highest weight vector $u_{+}$,

$$
\mathrm{e}^{2 \Lambda_{0}(\log (a(g))}=\left\langle g u_{+}, u_{+}\right\rangle, g \in \bar{N} L N
$$

In particular

$$
\bar{\nabla}(Y)^{2}=\mathrm{e}^{2 \Lambda_{0}\left(\log \left(a\left(w \bar{n}_{Y}\right)\right)\right)}=\left\langle w \bar{n}_{Y} u_{+}, u_{+}\right\rangle
$$

is a polynomial in $Y$. It follows that $\bar{\nabla}(Y)$ is defined on all of $\overline{\mathfrak{n}}$. 
As representation of $L, U_{1}^{\mathfrak{n}}$ (the $\mathfrak{n}$-invariants in $U_{1}$ ) is 1-dimensional and the $L$-action is by the character $\chi^{\frac{1}{d}}$ (which has differential $2 \Lambda_{0}$ on $\mathfrak{b}$ ). Also, $\left.\operatorname{Ad}(w)\right|_{\mathfrak{b}}=-1$ and $\chi(\theta(\ell))=\chi\left(\ell^{-1}\right)$. Now

$$
\begin{aligned}
& \bar{\nabla}(\ell \cdot Y)^{2}=\left\langle w \ell \bar{n}_{Y} \ell^{-1} u_{+}, u_{+}\right\rangle \\
& \quad=\left\langle w \ell w^{-1} w \bar{n}_{Y} \ell^{-1} u_{+}, u_{+}\right\rangle \\
& \quad=\chi\left(\ell^{-1}\right)^{\frac{1}{d}}\left\langle w \bar{n}_{Y} u_{+}, \theta\left(w \ell w^{-1}\right)^{-1} u_{+}\right\rangle \\
& \quad=\chi(\ell)^{-\frac{2}{d}}\left\langle w \bar{n}_{Y} u_{+}, u_{+}\right\rangle \\
& =\chi(\ell)^{-\frac{2}{d}} \bar{\nabla}(Y)^{2} \\
& \quad=\left(\mid \operatorname{det}\left(\left.\left.\operatorname{Ad}(\ell)\right|_{\bar{n}}\right|^{\frac{n}{m}} \bar{\nabla}(Y)\right)^{2} .\right.
\end{aligned}
$$

The corresponding statements for $\nabla$ follow.

Remark 2.11. The functions $\nabla$ are closely related to the determinant functions associated to the Jordan algebras; see Table 2 in Appendix A.

\section{INTEGRAL FORMULAS FOR THE ORBITS}

As in Section 2, write the $L$-orbits in $\mathfrak{n}$ as

$$
\mathcal{O}_{q}=L\left(X_{q}\right) \cong L / S_{q}, q=0,1,2, \ldots, n .
$$

The orbit $\mathcal{O}_{n}$ is open and dense in $\mathfrak{n}$, and is a semisimple symmetric space. In particular $\mathcal{O}_{n}$ has a unique (up to scalar multiple) invariant measure, which we denote by $\nu_{n}$. The semisimple symmetric space $L / S_{n}$ has a decomposition in terms of $K \cap L$ and $B \equiv \exp (\mathfrak{b})$. We note that $\mathfrak{b}$ is a Cartan subspace in $\mathfrak{l}$ perpendicular to both $\mathfrak{l} \cap \mathfrak{k}$ and $\mathfrak{s}_{n}$. Therefore, the Mostow decomposition is

$$
L=(K \cap L) B^{+} S_{n},
$$

with $B^{+}=\exp \left(\mathfrak{b}^{+}\right), \mathfrak{b}^{+}=\left\{H \in \mathfrak{b}: \alpha(H) \geq 0\right.$, for all $\left.\alpha \in \Sigma^{+}(\mathfrak{l}, \mathfrak{b})\right\}$. The invariant measure on $\mathcal{O}_{n}$ is of the form

$$
\int_{\mathcal{O}_{n}} F(X) d X=\int_{K \cap L} \int_{\mathfrak{b}^{+}} F\left(k b \cdot X_{n}\right) \delta(b) d b d k .
$$

Since $\mathcal{O}_{n}$ is open in $\mathfrak{n}$ we may express this measure in terms of Lebesgue measure $d X$ on $\mathfrak{n}$. The formula is

$$
\int_{\mathcal{O}_{n}} F(X) d \nu_{n}(X)=\int_{\mathfrak{n}} F(X) \nabla(X)^{-\frac{m}{n}} d X .
$$

This is easily verified since the action of $L$ on $\mathfrak{n}$ is linear.

The other orbits however do not have invariant measures. Instead, they have quasi-invariant measures $\nu_{q}$, $q=1,2, \ldots, n-1$. This means that for each $q$ there is a character $\tilde{\chi}: L \rightarrow \mathbf{R}^{\times}$so that

$$
\int_{\mathcal{O}_{q}} F(\ell \cdot X) d \nu_{q}(X)=\tilde{\chi}(\ell)^{-1} \int_{\mathcal{O}_{q}} F(X) d \nu_{q}(X) .
$$

In general a quasi-invariant measure $\nu$ on a homogeneous space $L / S$ corresponding to a character $\tilde{\chi}$ is determined by

$$
\int_{L / S} F_{f}(\ell S) d \nu(\ell S)=\int_{L} \tilde{\chi}(\ell) f(\ell) d \ell
$$

where $d \ell$ is a left invariant Haar measure on $L$ and

$$
F_{f}(\ell S)=\int_{S} f(\ell s) d s
$$


with $d s$ equal to left invariant Haar measure on $S$. Letting $\Xi$ denote the modular function for a Lie group, $\tilde{\chi}$-quasi-invariant measures exist if and only if $\tilde{\chi}$ is an extension of $\left.\Xi_{S} \Xi_{L}^{-1}\right|_{S}$ to a character of L. The modular function for a Lie group is $\Xi(\cdot)=|\operatorname{det}(\operatorname{Ad}(\cdot))|^{-1}$. See [14, Section 33] for details on quasi-invariant measures. In our situation $L$ is a reductive group, so its modular function is 1 . We need to compute the modular function of $S_{q}$.

We first describe the stabilizers $S_{q}=\operatorname{Stab}_{L}\left(X_{q}\right)$ in some detail. Let $\mathfrak{n}_{q}$ be the +2 -eigenspace of $\sum_{j=1}^{q} H_{j}$ in $\mathfrak{n}$. Then $\mathfrak{n}_{q}$ and $\overline{\mathfrak{n}}_{q} \equiv \theta\left(\mathfrak{n}_{q}\right)$ generate a semisimple Lie subalgebra $\mathfrak{g}_{q}$ for which the corresponding subgroup $G_{q}$ satisfies 2.1 ; we let $L_{q} N_{q}$ be the corresponding parabolic subgroup. Let $\mathfrak{b}_{q} \equiv \operatorname{span}_{\mathbf{R}}\left\{H_{1}, \ldots, H_{q}\right\}$. Then $\Sigma\left(\mathfrak{l}_{q}, \mathfrak{b}_{q}\right)=\left\{ \pm\left(\epsilon_{j}-\epsilon_{k}\right): 1 \leq j<k \leq q\right\}$. For each $q$ there is another subalgebra $\mathfrak{n}_{n-q}^{\prime}$ defined as the +2 -eigenspace of $\sum_{j=q+1}^{n} H_{j}$. The corresponding subalgebras are denoted by $\mathfrak{b}_{n-q}^{\prime}, \mathfrak{l}_{n-q}^{\prime}$ and $\overline{\mathfrak{n}}_{n-q}^{\prime}$. Then $\Sigma\left(\mathfrak{l}_{n-q}^{\prime}, \mathfrak{b}_{n-q}^{\prime}\right)=\left\{ \pm\left(\epsilon_{j}-\epsilon_{k}\right): q+1 \leq j<k \leq n\right\}$. The integers $d$ and $e$ for $\mathfrak{n}_{q}$ and $\mathfrak{n}_{n-q}^{\prime}$ are the same as for $\mathfrak{n}$, unless $q=1$ (resp. $q=n-1$ ) in which case $d=0$ for $\mathfrak{n}_{q}\left(\right.$ resp. $\left.\mathfrak{n}_{n-q}^{\prime}\right)$. We set $m_{q} \equiv \operatorname{dim}\left(\mathfrak{n}_{q}\right)$ and $m_{n-q}^{\prime}=\operatorname{dim}\left(\mathfrak{n}_{n-q}^{\prime}\right)$. Note that $\mathfrak{l}_{q}$ and $\mathfrak{l}_{n-q}^{\prime}$ commute. The following is straightforward to check.

Lemma 3.6. Let $S_{q}=\operatorname{Stab}_{L}\left(X_{q}\right)$ be the stabilizer in $L$ of $X_{q}$. Then the following statements hold.

(1) $Q_{q} \equiv\left\{\ell \in L: \ell \cdot \mathfrak{n}_{q} \subset \mathfrak{n}_{q}\right\}$ is a parabolic subgroup of $L$.

(2) $S_{q} \subset Q_{q}$.

(3) Setting $\mathcal{O}_{q}(q) \equiv L_{q}\left(X_{q}\right)$, the open orbit of $L_{q}$ in $\mathfrak{n}_{q}, Q_{q} / S_{q} \cong \mathcal{O}_{q}(q)$. The corresponding Mostow decomposition is $L_{q}=\left(K \cap L_{q}\right) B_{q}\left(S_{q} \cap L_{q}\right)$.

(4) Define $N_{q}^{L} \equiv \exp \left(\mathfrak{n}_{q}^{L}\right)$, where $\Sigma\left(\mathfrak{n}_{q}^{L}, \mathfrak{b}_{q}\right)=\left\{\epsilon_{i}-\epsilon_{j}: 1 \leq i \leq q<j \leq n\right\}$. Then $Q_{q}=L_{q} L_{n-q}^{\prime} N_{q}^{L}$ (with $N_{q}^{L}$ the nilradical) and $S_{q}=\left(L_{q} \cap S_{q}\right) L_{n-q}^{\prime} N_{q}^{L}$.

Corollary 3.7. The modular function $\Xi_{S_{q}}$ for $S_{q}$ has differential $2 d q \sum_{j=q+1}^{n} \epsilon_{j}$. Therefore $\Xi_{S_{q}}$ extends to the character $\chi_{q}$ on $L$; this is the character for the quasi-invariant measure on $\mathcal{O}_{q}$.

For $\mathfrak{n}_{q}$ (resp. $\mathfrak{n}_{n-q}^{\prime}$ ) the functions defined in $(2.9)$ are denoted by $\bar{\nabla}_{q}, \nabla_{q}$ (resp. $\bar{\nabla}_{n-q}^{\prime}, \nabla_{n-q}^{\prime}$ ).

The standard integration formula in terms of the Mostow decomposition $L_{q}=\left(K \cap L_{q}\right) B_{q}\left(S_{q} \cap L_{q}\right)$ (see $(3.2))$ is

$$
\int_{\mathcal{O}_{q}(q)} F(X) d \nu_{q}^{q}(X)=\int_{K \cap L_{q}} \int_{B_{q}^{+}} F\left(k b \cdot E_{q}\right) \delta_{q}(b) d b d k .
$$

The exact form of $\delta_{q}$, which is not needed here, is given in [23, Section 8.1]. An invariant measure $\nu_{q}^{q}$ on $\mathcal{O}_{q}(q)$ may also be given in terms of Lebesgue measure on $\mathfrak{n}_{q}$ (as in (3.3)) by

$$
\int_{\mathcal{O}_{q}(q)} F(X) d \nu_{q}^{q}(X)=\int_{\mathcal{O}_{q}(q)} F(X) \nabla_{q}(X)^{-\frac{m_{q}}{q}} d X .
$$

In the decomposition $Q_{q}=L_{q} L_{n-q}^{\prime} N_{q}^{L}$ we may write $L_{q}=M_{q} A_{q}$ and $L_{n-q}^{\prime}=M_{n-q}^{\prime} A_{n-q}^{\prime}$ so that the Langlands decomposition of $Q_{q}$ is $M_{q} M_{n-q}^{\prime} A_{q} A_{n-q}^{\prime} N_{q}^{L}$.

Lemma 3.9. For $q=1,2, \ldots, n-1$ the following formula gives a $\chi_{q}$-quasi invariant measure on $\mathcal{O}_{q}$.

$$
\int_{\mathcal{O}_{q}} F(X) d \nu_{q}(X)=\int_{K \cap L} \int_{B_{q}^{+}} F\left(k b \cdot E_{q}\right) \chi_{n}(b) \delta_{q}(b) d b d k .
$$


Proof. The standard integration formula for a group in terms of a parabolic subgroup gives us

$$
\begin{aligned}
& \int_{L} \chi_{q}(\ell) f(\ell) d \ell \\
& \quad=\int_{K \cap L} \int_{M_{q} M_{n-q}^{\prime} A_{q} A_{n-q}^{\prime}} \int_{N_{q}^{L}} \chi_{q}\left(a a^{\prime}\right) f\left(k m m^{\prime} a a^{\prime}\right) \mathrm{e}^{2 \rho\left(\mathfrak{n}_{q}^{L}\right)}\left(a a^{\prime}\right) d n d a d a^{\prime} d m d m^{\prime} d k
\end{aligned}
$$

Since $2 \rho\left(\mathfrak{n}_{q}^{L}\right)=2 d\left((n-q) \sum_{1}^{q} \epsilon_{j}-q \sum_{q+1}^{n} \epsilon_{j}\right)$ we have $\chi_{q}\left(a a^{\prime}\right) e^{2 \rho\left(\mathfrak{n}_{q}^{L}\right)}\left(a a^{\prime}\right)=e^{2 d n \Lambda_{0, q}}(a)$, for $\Lambda_{0, q}=\sum_{j=1}^{q} \epsilon_{j}$. Thus,

$$
\begin{aligned}
\int_{L} & \chi_{q}(\ell) f(\ell) d \ell \\
& =\int_{K \cap L} \int_{M_{q} A_{q}} \int_{M_{n-q}^{\prime} A_{n-q}^{\prime}} \int_{N_{q}^{L}} f\left(k m a m^{\prime} a^{\prime} n\right) \mathrm{e}^{2 d n \Lambda_{0, q}}(a) d n d a d a^{\prime} d m d m^{\prime} d k \\
& =\int_{K \cap L} \int_{M_{n-q}^{\prime} A_{n-q}^{\prime}} \int_{M_{q} A_{q} / M_{q} A_{q} \cap S_{q}} \int_{M_{q} A_{q} \cap S_{q}} \int_{N_{q}^{L}} f\left(k m a m^{\prime} a^{\prime} n\right) \mathrm{e}^{2 d n \Lambda_{0, q}}(a) d n d a d a^{\prime} d m d m^{\prime} d k \\
& =\int_{L \cap K} \int_{M_{n-q}^{\prime} A_{n-q}^{\prime}} \int_{S_{q}} f(k m a s) \mathrm{e}^{2 d n \Lambda_{0, q}} d s d m d a d k \\
& =\int_{K \cap L} \int_{K \cap L_{q}} \int_{B_{q}^{+}} \int_{S_{q}} f\left(k k_{1} b s\right) \mathrm{e}^{2 d n \Lambda_{0, q}}(b) \delta_{q}(b) d s d b d k_{1} d k \\
& =\int_{K \cap L} \int_{B_{q}^{+}} F_{f}(k b) \chi_{q}(b)^{\frac{n}{q}} \delta_{q}(b) d b d k .
\end{aligned}
$$

The lemma follows from (3.5).

Inserting the integration formula (3.8) for the dense orbit $\mathcal{O}_{q}(q) \subset \mathfrak{n}_{q}$ we obtain the following useful formula. We remark that normalizations of the Lebesgue measures on $\mathfrak{n}, \overline{\mathfrak{n}}$ and subspaces, and on $\mathcal{O}_{q}$ have not yet been given. We give normalizations of the Lebesgue measures just before Prop. 3.13 and the normalization of $\nu_{q}$ will be given in (5.3).

Corollary 3.10. For $q=1,2, \ldots, n-1$

$$
\int_{\mathcal{O}_{q}} F(X) d \nu_{q}(X)=\int_{K \cap L} \int_{\mathfrak{n}_{q}} F(k \cdot Y) \nabla_{q}(Y)^{d n-\frac{m_{q}}{q}} d Y d k .
$$

Proof. Note that $\chi_{n}(k b)=\nabla_{q}\left(k b \cdot X_{q}\right)^{d n}$. We therefore have

$$
\begin{array}{rl}
\int_{\mathcal{O}_{q}} & F(X) d \nu_{q}(X) \\
& =\int_{K \cap L} \int_{B_{q}^{+}} F\left(k b \cdot X_{q}\right) \chi_{n}(b) \delta_{q}(b) d b d k \\
& =\int_{K \cap L} \int_{\mathcal{O}_{q}(q)} F(k \cdot Y) \nabla_{q}(Y)^{n d} \nabla_{q}(Y)^{-\frac{m_{q}}{q}} d Y d k
\end{array}
$$

Since $\mathcal{O}_{q}(q)$ is dense in $\mathfrak{n}_{q}$ we may integrate over $\mathfrak{n}_{q}$.

Corollary 3.11. Let $\overline{\mathfrak{n}}_{q}^{L}=\theta\left(\mathfrak{n}_{q}^{L}\right)$. Then

$$
\int_{\mathcal{O}_{q}} F(X) d \nu_{q}(X)=C \int_{\overline{\mathfrak{n}}_{q}^{L}} \int_{\mathfrak{n}_{q}} F(\exp (u) \cdot X) \nabla_{q}(X)^{n d-\frac{m_{q}}{q}} d X d u
$$

Proof. We convert the integral over $K \cap L$ in the preceding corollary into an integral over $\overline{\mathfrak{n}}_{q}^{L}$ using [10, Eq. 5.25]. We write the 'Iwasawa' decomposition of $\exp (u), u \in \overline{\mathfrak{n}}_{q}^{L}$ with respect to the parabolic $Q_{q}$ as 
$\exp (u)=\kappa(\exp (u)) m_{u} \exp \left(H(\exp (u)) n_{u} \in K M_{q} A_{q} N_{q}\right.$. Then

$$
\begin{array}{rl}
\int_{\mathcal{O}_{q}} & F(X) d \nu_{q}(X) \\
& =\int_{\overline{\mathfrak{n}}_{q}^{L}} \int_{\mathfrak{n}_{q}} F\left(\kappa(\exp (u) \cdot X) \nabla_{q}(X)^{n d-\frac{m_{q}}{q}} \mathrm{e}^{-2 \rho\left(\overline{\mathfrak{n}}_{q}^{L}\right)(H(\exp (u)))} d X d u\right. \\
& =\int_{\overline{\mathfrak{n}}_{q}^{L}} \int_{\mathfrak{n}_{q}} F\left(\exp (u) \exp (H(\exp (u)))^{-1} m_{u}^{-1} n_{u}^{-1} \cdot X\right) \nabla_{q}(X)^{n d-\frac{m_{q}}{q}} \mathrm{e}^{-2 \rho\left(\overline{\mathfrak{n}}_{q}^{L}\right)(H(\exp (u)))} d X d u
\end{array}
$$

Note that $\exp (H(\exp (u)))^{-1} m_{u}^{-1} n_{u}^{-1}$ stabilizes $\mathfrak{n}_{q}$. Therefore, by Lemma 2.10 the result of the change of variables $X \mapsto \exp (H(\exp (u)))^{-1} m_{u}^{-1} n_{u}^{-1} \cdot X$ on $\mathfrak{n}_{q}$ is

$$
\begin{array}{r}
\int_{\overline{\mathfrak{n}}_{q}^{L}} \int_{\mathfrak{n}_{q}} F(\exp (u) \cdot X) \nabla_{q}(X)^{n d-\frac{m_{q}}{q}}\left(\operatorname{det}\left(\operatorname{Ad}\left(\left.\mathrm{e}^{H(\exp (u))}\right|_{\mathfrak{n}_{q}}\right)^{\frac{q}{m_{q}}}\right)^{n d-\frac{m_{q}}{q}}\right. \\
\mathrm{e}^{-2 \rho\left(\overline{\mathfrak{n}}_{q}^{L}\right)(H(\exp (u)))} \operatorname{det}\left(\operatorname{Ad}\left(\left.\mathrm{e}^{H(\exp (u))}\right|_{\mathfrak{n}_{q}}\right) d X d u .\right.
\end{array}
$$

Claim: The terms involving $H(\exp (u))$ cancel out. This follows from

$$
\begin{gathered}
\left(\operatorname { d e t } ( \operatorname { A d } ( \mathrm { e } ^ { H ( \operatorname { e x p } ( u ) ) } | _ { \mathfrak { n } _ { q } } ) ^ { \frac { q } { m _ { q } } } ) ^ { n d - \frac { m _ { q } } { q } } \mathrm { e } ^ { - 2 \rho ( \overline { \mathfrak { n } } _ { q } ^ { L } ) ( H ( \operatorname { e x p } ( u ) ) ) } \operatorname { d e t } \left(\operatorname{Ad}\left(\left.\mathrm{e}^{H(\exp (u))}\right|_{\mathfrak{n}_{q}}\right)\right.\right. \\
=\mathrm{e}^{2 q d \Lambda_{0}(H(\exp (u)))}=\chi_{q}(\exp (H(\exp (u))))=1,
\end{gathered}
$$

since $H(\exp (u))$ is in the semisimple part of $\mathfrak{l}(\operatorname{as} \exp (u)$ is $)$.

We will need another integration formula. This one relates Lebesgue measure on $\mathfrak{n}$ to Lebesgue measures on $\overline{\mathfrak{n}}_{q}^{L}, \mathfrak{n}_{q}$ and $\mathfrak{n}_{n-q}^{\prime}$. We take care in normalizing these measures as we wish to obtain exact formulas later.

Let $B_{\mathfrak{g}}$, or simply $B$, denote the Killing form of $\mathfrak{g}$. Then $B$ gives a nondegenerate pairing between $\mathfrak{n}$ and $\overline{\mathfrak{n}}$. Recall that $m$ is the dimension of $\mathfrak{n}$. Set

$$
\langle,\rangle_{\mathfrak{g}} \equiv \frac{n}{4 m} B_{\mathfrak{g}}
$$

giving a nondegenerate pairing between $\mathfrak{n}$ and $\overline{\mathfrak{n}}$.

Fact : The restriction of $\langle,\rangle_{\mathfrak{g}}$ to $\mathfrak{g}_{q} \times \mathfrak{g}_{q}$ is $\langle,\rangle_{\mathfrak{g}_{q}}$. To see this note that, since $\mathfrak{g}_{q}$ is simple and $\langle,\rangle_{\mathfrak{g}}$ is $\mathfrak{g}_{q}$-invariant, there is a nonzero constant $c$ so that $\langle,\rangle_{\mathfrak{g}}=c\langle,\rangle_{\mathfrak{g}_{q}}$ on $\mathfrak{g}_{q} \times \mathfrak{g}_{q}$. To see that $c=1$ compute

$$
\left\langle H_{1}, H_{1}\right\rangle_{\mathfrak{g}}=\operatorname{trace}\left(a d\left(H_{1}\right)^{2}\right)=\frac{n}{4 m} 8(d(n-1)+(e+1))=2,
$$

(since $m=n(d(n-1)+(e+1)))$ and

$$
\left\langle H_{1}, H_{1}\right\rangle_{\mathfrak{g}_{q}}=\operatorname{trace}\left(\left.\operatorname{ad}\left(H_{1}\right)^{2}\right|_{\mathfrak{g}_{q}}\right)=\frac{q}{4 m_{q}} 8(d(q-1)+(e+1))=2 .
$$

We will use $\langle$,$\rangle to denote \langle,\rangle_{\mathfrak{g}}$ for any $\mathfrak{g}$. The significance of the above fact is that when we pass from $\mathfrak{n}$ to $\mathfrak{n}_{q}$ in the induction arguments below the pairing $\langle$,$\rangle is unchanged.$

As the form $-B(\cdot, \theta(\cdot))$ is positive definite on $\mathfrak{g},\langle$,$\rangle defines a inner products on \mathfrak{n}$. Denote this inner product by $\left\langle X_{1}, X_{2}\right\rangle_{\theta}=-\left\langle X_{1}, \theta\left(X_{2}\right)\right\rangle$. The corresponding norms

$$
\|X\|^{2}=\langle X, X\rangle_{\theta}=-\langle X, \theta(X)\rangle, X \in \mathfrak{n}, \quad \text { and } \quad\|Y\|^{2}=\langle Y, Y\rangle_{\theta}=-\langle Y, \theta(Y)\rangle, Y \in \overline{\mathfrak{n}} .
$$

determine Lebesgue measures $d X$ on $\mathfrak{n}$ and $d Y$ on $\overline{\mathfrak{n}}$ in a standard way.

Define

$$
\begin{aligned}
& \tilde{\mathfrak{n}}=\sum \mathfrak{g}_{\epsilon_{j}+\epsilon_{k}}, \quad 1 \leq j \leq q<k \leq n, \text { and } \\
& \overline{\widetilde{\mathfrak{n}}}=\sum \mathfrak{g}_{-\left(\epsilon_{j}+\epsilon_{k}\right)}, \quad 1 \leq j \leq q<k \leq n .
\end{aligned}
$$


The inner product $\langle,\rangle_{\theta}$ is nondegenerate on each of $\widetilde{\mathfrak{n}}, \overline{\mathfrak{n}}$ and $\overline{\mathfrak{n}}_{q}^{L}$. Give $\widetilde{\mathfrak{n}}, \overline{\tilde{\mathfrak{n}}}$ and $\overline{\mathfrak{n}}_{q}^{L}$ the Lebesgue measures determined by the restriction of $\langle,\rangle_{\theta}$ to each subspace.

Lemma 3.12. For $X \in \mathfrak{n}_{q}$ (resp. $\left.Y^{\prime} \in \overline{\mathfrak{n}}_{n-q}^{\prime}\right)$ with $\nabla_{q}(X) \neq 0\left(\operatorname{resp} . \bar{\nabla}_{n-q}\left(Y^{\prime}\right) \neq 0\right) \operatorname{ad}(X): \overline{\mathfrak{n}}_{q}^{L} \rightarrow \widetilde{\mathfrak{n}}(\operatorname{resp}$. $\operatorname{ad}\left(Y^{\prime}\right): \overline{\mathfrak{n}}_{q}^{L} \rightarrow \overline{\tilde{\mathfrak{n}}}$ is invertible. For $\nabla_{q}(X) \neq 0$ and $H \in L^{1}(\widetilde{\mathfrak{n}})$

$$
\nabla_{q}(X)^{2 d(n-q)} \int_{\tilde{\mathfrak{n}}} H(z) d z=\int_{\overline{\mathfrak{n}}_{q}^{L}} H(a d(X) u) d u
$$

and for $\bar{\nabla}_{n-q}\left(Y^{\prime}\right) \neq 0$ and $F \in L^{1}(\overline{\widetilde{\mathfrak{n}}})$

$$
\bar{\nabla}_{n-q}\left(Y^{\prime}\right)^{2 d q} \int_{\widetilde{\mathfrak{n}}} F\left(z^{\prime}\right) d z^{\prime}=\int_{\overline{\mathfrak{n}}_{q}^{L}} F\left(a d\left(Y^{\prime}\right) u\right) d u
$$

Proof. Suppose $X \in \mathfrak{n}_{q}$ and $\nabla_{q}(X) \neq 0$. We may write $X=\ell \cdot X_{q}, X_{q}=\sum_{j=1}^{q} E_{j}$. Fix orthonormal bases of $\widetilde{\mathfrak{n}}$ and $\overline{\mathfrak{n}}_{q}^{L}$. We compute the determinant of $T \equiv \operatorname{ad}(X): \overline{\mathfrak{n}}_{q}^{L} \rightarrow \widetilde{\mathfrak{n}}$ with respect to these bases. First we check that $T \equiv \operatorname{ad}\left(X_{q}\right): \overline{\mathfrak{n}}_{q}^{L} \rightarrow \widetilde{\mathfrak{n}}$ preserves inner products (so has determinant 1 ).

Observe that

$$
\operatorname{ad}\left(\theta X_{q}\right) \operatorname{ad}\left(X_{q}\right) u=\left[\theta X_{q},\left[X_{q}, u\right]\right]=\left[u,\left[\theta X_{q}, X_{q}\right]\right]=\sum_{j=1}^{q}\left[H_{j}, u\right]=-u,
$$

therefore, $-\operatorname{ad}\left(\theta X_{q}\right) \operatorname{ad}\left(X_{q}\right)$ is the identity on $\overline{\mathfrak{n}}_{q}^{L}$. Now

$$
\begin{aligned}
\left\langle\operatorname{ad}\left(X_{q}\right) u\right. & \left., \operatorname{ad}\left(X_{q}\right) v\right\rangle_{\theta}=\left\langle\operatorname{ad}\left(X_{q}\right) u, \theta\left(\operatorname{ad}\left(X_{q}\right) v\right)\right\rangle \\
& =\left\langle-\operatorname{ad}\left(\theta\left(X_{q}\right)\right) \operatorname{ad}\left(X_{q}\right) u, \theta(v)\right\rangle \\
& =\langle u, v\rangle_{\theta}
\end{aligned}
$$

We can now calculate the determinant of $T$ with respect to the orthonormal bases of $\overline{\mathfrak{n}}_{q}^{L}$ and $\widetilde{\mathfrak{n}}$.

$$
\begin{aligned}
\operatorname{det}(T) & =\left.\operatorname{det}\left(\left.\operatorname{Ad}(\ell)\right|_{\mathfrak{n}}\right) \operatorname{det}\left(\operatorname{ad}(X): \overline{\mathfrak{n}}_{q}^{L} \rightarrow \widetilde{\mathfrak{n}}\right) \operatorname{det}\left(\operatorname{Ad}\left(\ell^{-1}\right)\right)\right|_{\overline{\mathfrak{n}}_{q}^{L}} \\
& =\chi(\ell)^{n-q} \cdot 1 \cdot \chi\left(\ell^{-1}\right)^{-(n-q)} \\
& =\chi(\ell)^{2(n-q)} \\
& =\nabla_{q}(X)^{2 d(n-q)} .
\end{aligned}
$$

Similarly, for $\bar{\nabla}_{n-q}\left(Y^{\prime}\right) \neq 0, \operatorname{ad}\left(Y^{\prime}\right): \overline{\mathfrak{n}}_{q}^{L} \rightarrow \overline{\tilde{\mathfrak{n}}}$ is an isometry and $\operatorname{det}\left(\operatorname{ad}\left(Y^{\prime}\right): \overline{\mathfrak{n}}_{q}^{L} \rightarrow \overline{\widetilde{\mathfrak{n}}}\right)=\bar{\nabla}_{n-q}\left(Y^{\prime}\right)^{2 d q}$.

Proposition 3.13. Let $f \in L^{1}(\mathfrak{n})$ and $h \in L^{1}(\overline{\mathfrak{n}})$. Then

$$
\begin{aligned}
\int_{\mathfrak{n}} f(X) d X & =\int_{\overline{\mathfrak{n}}_{q}^{L}} \int_{\mathfrak{n}_{q}} \int_{\mathfrak{n}_{n-q}^{\prime}} f\left(\exp (u) \cdot\left(X+X^{\prime}\right)\right) \nabla_{q}(X)^{2 d(n-q)} d X d X^{\prime} d u \\
\int_{\overline{\mathfrak{n}}^{\prime}} h(Y) d Y & =\int_{\overline{\mathfrak{n}}_{q}^{L}} \int_{\overline{\mathfrak{n}}_{q}} \int_{\overline{\mathfrak{n}}_{n-q}^{\prime}} h\left(\exp (u) \cdot\left(Y+Y^{\prime}\right)\right) \bar{\nabla}_{n-q}\left(Y^{\prime}\right)^{2 d q} d Y d Y^{\prime} d u .
\end{aligned}
$$

Proof. We prove only the first formula, the second is proved by essentially the same argument. Since $\bar{Q}_{q} S_{q}=\bar{N}_{q}^{L} Q_{q}$ is dense in $L$ and the complement is of measure zero,

$$
\bar{N}_{q}^{L} L_{q} L_{n-q}^{\prime}\left(X_{q}+X_{n-q}^{\prime}\right) \subset \bar{N}_{q}^{L} \cdot\left(\mathfrak{n}_{q}+\mathfrak{n}_{n-q}^{\prime}\right)
$$

have full measure in $\mathfrak{n}$. We compute the Jacobian of the transformation

$$
\begin{aligned}
& \phi: \mathfrak{n}_{q} \times \overline{\mathfrak{n}}_{q}^{L} \times \mathfrak{n}_{n-q}^{\prime} \rightarrow \mathfrak{n} \\
& \left(X, u, X^{\prime}\right) \mapsto \exp (u) \cdot\left(X+X^{\prime}\right)
\end{aligned}
$$


and see that $\phi$ is a diffeomorphism on the open subset of $\left(X, u, X^{\prime}\right) \in \mathfrak{n}_{q} \times \overline{\mathfrak{n}}_{q}^{L} \times \mathfrak{n}_{n-q}^{\prime}$ where $\nabla_{q}(X) \neq 0$.

Note that $\mathfrak{n}=\mathfrak{n}_{q}+\widetilde{\mathfrak{n}}+\mathfrak{n}_{n-q}^{\prime}$. The matrix of the differential of $\phi$ at $\left(X, u, X^{\prime}\right)$ with respect to orthonormal bases of $\mathfrak{n}_{q}, \overline{\mathfrak{n}}_{q}^{L}, \mathfrak{n}_{n-q}^{\prime}$ and $\mathfrak{n}_{q}, \widetilde{\mathfrak{n}}, \mathfrak{n}_{n-q}^{\prime}$ has the form

$$
\left(\begin{array}{lll}
I & 0 & 0 \\
* & T & 0 \\
* & * & I
\end{array}\right)
$$

where $T=\operatorname{ad}(X): \overline{\mathfrak{n}}_{q}^{L} \rightarrow \widetilde{\mathfrak{n}}$. Therefore the Jacobian is $\operatorname{det}(T)=\nabla_{q}(X)^{2 d(n-q)}$ as computed in Lemma 3.12 .

The following lemma, which is essentially only a restatement of the of what is proved above, will be used in Sections 4 and 5 Let $\pi_{q}: \mathfrak{n} \rightarrow \mathfrak{n}_{q}$ be the orthogonal projection (with respect to $\langle,\rangle_{\theta}$ ). Set $\mathcal{U}_{q}=\{X \in \mathfrak{n}$ : $\left.\nabla_{q}(X) \neq 0\right\}$, an open set in $\mathfrak{n}$.

Lemma 3.14. For $X \in \mathfrak{n}_{q}$ with $\nabla_{q}(X) \neq 0, \operatorname{ad}(X): \overline{\mathfrak{n}}_{q}^{L} \rightarrow \widetilde{\mathfrak{n}}$ is invertible. The change of coordinates $\phi:\left(X, u, X^{\prime}\right) \mapsto \exp (u)\left(X+X^{\prime}\right)$ is a smooth one-to-one map $\mathfrak{n}_{q} \times \overline{\mathfrak{n}}_{q}^{L} \times \mathfrak{n}_{n-q}^{\prime} \rightarrow \mathfrak{n}$ and is a diffeomorphism from $\mathcal{O}_{q}(q) \times \overline{\mathfrak{n}}_{q}^{L} \times \mathfrak{n}_{n-q}^{\prime}$ onto $\mathcal{U}_{q}$. The Jacobian is $\nabla_{q}(X)^{2 d(n-q)}$.

Proof. The Jacobian is computed in the proof of Lemma 3.13. To see that $\phi$ is a bijection $\mathcal{O}_{q}(q) \times \overline{\mathfrak{n}}_{q}^{L} \times \mathfrak{n}_{n-q}^{\prime} \rightarrow$ $\mathcal{U}_{q}$ write $\exp (u)\left(X+X^{\prime}\right)=X+\operatorname{ad}(u) X+\left(X^{\prime}+\frac{1}{2} \operatorname{ad}(u)^{2}(X)\right) \in \mathfrak{n}_{q}+\widetilde{\mathfrak{n}}+\mathfrak{n}_{n-q}^{\prime}$ and use the fact that $\operatorname{ad}(X)$ is invertible when $\nabla_{q}(X) \neq 0$.

We end this section with two facts we will need later.

Lemma 3.15. For $\alpha \in \mathbf{C}$

$$
\int_{\overline{\mathfrak{n}}} e^{-\alpha \Lambda_{0}\left(H\left(\bar{n}_{Y}\right)\right)} d Y
$$

is finite for $\operatorname{Re}(\alpha)>\frac{m}{n}+d(n-1)$.

Proof. This is a standard argument. See [10, Cor. 7.7].

We are also interested in the local integrability of powers of $\nabla(X)$ and $\bar{\nabla}(Y)$. For this we apply the standard integration formula for a semisimple symmetric space (as in equation (3.2)). The invariant measure in the polar coordinates $(K \cap L) B^{+}$is of the form $\delta(b) d b d k$. It follows from [23, page 149] that $|\delta(b)| \leq$ $\mathrm{e}^{2 \rho(\mathfrak{l})}(b)$.

Lemma 3.16. For $\operatorname{Re}(\alpha)>-(e+1)$ the function $\nabla(X)^{\alpha}$ (resp. $\left.\bar{\nabla}(Y)^{\alpha}\right)$ is a locally $L_{1}$ function on $\mathfrak{n}$ (resp. n) and defines a tempered distribution.

Proof. We use the polar coordinates $(K \cap L) B^{+}$and formulas (3.2) and (3.3) to check that

$$
\int_{\mathfrak{b}_{c}^{+}} \nabla\left(b_{t} \cdot X_{n}\right)^{\operatorname{Re}(\alpha)+\frac{m}{n}} \delta\left(b_{t}\right) d t<\infty,
$$

where

$$
\mathfrak{b}_{c}^{+}=\left\{t \in \mathbf{R}^{n}: c>t_{1}>t_{2} \cdots\right\}
$$

and

$$
b_{t}=\exp \left(\sum_{j=1}^{n} t_{j} H_{j}\right)
$$


By Lemma $2.10(\mathrm{~b})$

$$
\nabla\left(b_{t} \cdot X_{n}\right)=\prod_{j=1}^{n} \mathrm{e}^{2 t_{j}}
$$

Now

$$
\int_{\mathfrak{b}_{c}^{+}} \nabla\left(b_{t} \cdot X_{n}\right)^{\operatorname{Re}(\alpha)+\frac{m}{n}} \mathrm{e}^{2 \rho(\mathfrak{l})} d t=\int_{c>t_{1}>\cdots>t_{n}} \prod_{j} \mathrm{e}^{\left(\operatorname{Re}(\alpha)+\frac{m}{n}\right) 2 t_{j}+2 d(n-2 j+1) t_{j}} d t_{1} \ldots d t_{n}<\infty
$$

for $\operatorname{Re}(\alpha)+\frac{m}{n}+d(n-2 j+1)>0$ for $j=0,1, \ldots, n$, i.e., $\operatorname{Re}(\alpha)>-\frac{m}{n}+d(n-1)=-(e+1)$.

\section{Functional equation}

The functions $\nabla(X)^{s}$ and $\bar{\nabla}(Y)^{s}$ are locally $L_{1}$ functions for $\operatorname{Re}(s)>-(e+1)$ by Lemma 3.16. Therefore tempered distributions are defined by the integrals

$$
\mathbf{Z}(h, s)=\int_{\mathfrak{n}} h(X) \nabla(X)^{s} d X, \text { for } h \in \mathcal{S}(\mathfrak{n}) \text { and } \overline{\mathbf{Z}}(f, s)=\int_{\overline{\mathfrak{n}}} f(X) \bar{\nabla}(X)^{s} d X, \text { for } f \in \mathcal{S}(\overline{\mathfrak{n}}) .
$$

Here $\mathcal{S}(\mathfrak{n})$ (resp. $\mathcal{S}(\overline{\mathfrak{n}})$ ) denotes the space of Schwartz functions on $\mathfrak{n}$ (resp. $\overline{\mathfrak{n}}$ ). Note that in the range $\operatorname{Re}(s)>-(e+1)$ both expressions are complex analytic functions of $s$. We will see that there is a meromorphic continuation to all of $\mathbf{C}$ and a functional equation relating the two distributions via the Fourier transform.

The fact that there is a meromorphic continuation is well-know ([21], [25] and [22]). The explicit functional equation (Theorem 4.4) below has been studied in various forms. See [15] and [4]. Theorem 4.4 below computes the coefficients which occur in the functional equation and is a special case of Proposition 3 in [15]. When $G$ is a complex group it is contained in [3, Theorem 3.16]. Most of the statements in this section are contained in [15]; we include the details of the proofs since we will need much of the setup and many of the formulas which arise.

By Lemma $2.10 P(X) \equiv \nabla(X)^{2}$ and $\bar{P}(Y) \equiv \bar{\nabla}(Y)^{2}$ are polynomials. They define constant coefficient differential operators characterized by

$$
P\left(\partial_{X}\right) \mathrm{e}^{\langle X, Y\rangle}=\bar{P}(Y) \mathrm{e}^{\langle X, Y\rangle} \text { and } \bar{P}\left(\partial_{Y}\right) \mathrm{e}^{\langle X, Y\rangle}=P(X) \mathrm{e}^{\langle X, Y\rangle} .
$$

There is a polynomial $b(s)([2])$ so that

$$
P\left(\partial_{X}\right) \nabla(X)^{s}=b(s) \nabla(X)^{s-2} \text { and } \bar{P}\left(\partial_{Y}\right) \bar{\nabla}(Y)^{s}=b(s) \bar{\nabla}(Y)^{s-2} .
$$

In particular, for $b_{k}(s) \equiv b(s) b(s-2) b(s-4) \cdots b(s-2(k-1))$,

$$
P\left(\partial_{X}\right)^{k} \nabla(X)^{s}=b_{k}(s) \nabla(X)^{s-2 k} \text { and } \bar{P}\left(\partial_{Y}\right)^{k} \bar{\nabla}(Y)^{s}=b_{k}(s) \bar{\nabla}(y)^{s-2 k} \text {. }
$$

It follows that

$$
\mathbf{Z}\left(P\left(\partial_{X}\right)^{k} h, s\right)=b_{k}(s) \mathbf{Z}(h, s-2 k)
$$

and

$$
\overline{\mathbf{Z}}\left(\bar{P}\left(\partial_{Y}\right)^{k} f, s\right)=b_{k}(s) \overline{\mathbf{Z}}(f, s-2 k)
$$

for $\operatorname{Re}(s) \gg 0$. Since the left hand side is analytic for $\operatorname{Re}(s)>-(e+1), \mathbf{Z}(h, s)$ and $\overline{\mathbf{Z}}(f, s)$ continue to meromorphic functions on $\operatorname{Re}(s)>-(e+1)-2 k$ for any $k$. Let $\left\{\alpha_{j}\right\}$ be the set of roots of $b(s)$ and set $S=\left\{\alpha_{j}-2 l: l \in \mathbf{Z}_{+}\right\}$. We may conclude the following lemma.

Lemma 4.3. For $h \in \mathcal{S}(\mathfrak{n})$ (resp. $f \in \mathcal{S}(\overline{\mathfrak{n}})) Z(h, s)$ (resp. $\overline{\mathbf{Z}}(f, s)$ ) has a meromorphic continuation with $S$ the set of potential poles. 
We now turn to the Fourier transform and functional equation. Recall the definition of the pairing $\langle$, given in Section 3. Define the Fourier transforms by

$$
\widehat{h}(Y)=\int h(X) \mathrm{e}^{-2 \pi i\langle Y, X\rangle} d X, \text { for } h \in \mathcal{S}(\mathfrak{n})
$$

and

$$
\widehat{f}(X)=\int f(Y) \mathrm{e}^{-2 \pi i\langle Y, X\rangle} d Y, \text { for } f \in \mathcal{S}(\overline{\mathfrak{n}}) .
$$

The normalization of Lebesgue measure is so that Fourier inversion is

$$
\widehat{\widehat{h}}(X)=h(-X) \text { and } \widehat{\widehat{f}}(Y)=f(-Y) \text {. }
$$

The main result of this section is the following theorem. We let $\Gamma$ denote the gamma function on $\mathbf{C}$.

Theorem 4.4. ([15]) Let $s \in \mathbf{C}$ and $f \in \mathcal{S}(\overline{\mathfrak{n}})$. As meromorphic functions

$$
\frac{\pi^{\frac{n s}{2}}}{\Gamma_{n}(s)} \mathbf{Z}\left(\widehat{f}, s-\frac{m}{n}\right)=\frac{\pi^{\frac{n}{2}\left(-s+\frac{m}{n}\right)}}{\Gamma_{n}\left(-s+\frac{m}{n}\right)} \overline{\mathbf{Z}}(f,-s)
$$

where

$$
\Gamma_{n}(s) \equiv \prod_{j=0}^{n-1} \Gamma\left(\frac{s-j d}{2}\right) .
$$

We begin the proof with a few preliminary propositions. The first step is Weil's integration formula ([27]). Recall the subalgebras of $\mathfrak{g}$ defined in Section 3:

$$
\begin{aligned}
& \mathfrak{n}_{q}=\sum \mathfrak{g}_{\epsilon_{i}+\epsilon_{j}}, \text { for } 1 \leq i, j \leq q \\
& \mathfrak{n}_{n-q}^{\prime}=\sum \mathfrak{g}_{\epsilon_{i}+\epsilon_{j}}, \text { for } q<i, j \leq n \\
& \overline{\mathfrak{n}}_{q}=\sum \mathfrak{g}_{-\epsilon_{i}-\epsilon_{j}} \text { for } 1 \leq i, j \leq q \\
& \overline{\mathfrak{n}}_{n-q}^{\prime}=\sum \mathfrak{g}_{-\epsilon_{i}-\epsilon_{j}} \text { for } q<i, j \leq \\
& \overline{\mathfrak{n}}_{q}^{L}=\sum \mathfrak{g}_{-\epsilon_{i}+\epsilon_{j}}, \text { for } 1 \leq i \leq q<j \leq n \\
& \widetilde{\mathfrak{n}}=\sum \mathfrak{g}_{\epsilon_{i}+\epsilon_{j}}, \text { for } 1 \leq i \leq q<j \leq n \\
& \overline{\mathfrak{n}}=\sum \mathfrak{g}_{-\epsilon_{i}-\epsilon_{j}} \text { for } 1 \leq i \leq q<j \leq n .
\end{aligned}
$$

Observe that for $u \in \overline{\mathfrak{n}}_{q}^{L}$

$$
\begin{aligned}
& \operatorname{ad}(u): \mathfrak{n}_{q} \rightarrow \widetilde{\mathfrak{n}}, \\
& \operatorname{ad}(u): \widetilde{\mathfrak{n}} \rightarrow \mathfrak{n}_{n-q}^{\prime}, \\
& \operatorname{ad}(u): \mathfrak{n}_{n-q}^{\prime} \rightarrow 0 .
\end{aligned}
$$

Therefore,

$$
\operatorname{Ad}\left(\exp ((u))=I+a d(u)+\frac{1}{2}(a d(u))^{2}\right.
$$

on $\mathfrak{n}_{q}$ and

$$
\operatorname{ad}(u)^{2}: \mathfrak{n}_{q} \rightarrow \mathfrak{n}_{n-q}^{\prime}
$$

Define the Fourier transform on $\overline{\mathfrak{n}}_{q}^{L}$ by

$$
\widehat{\phi}(v)=\int_{\overline{\mathfrak{n}}_{q}^{L}} \phi(u) \mathrm{e}^{-2 \pi i\langle u, v\rangle_{\theta}} d u
$$

for $\phi \in \mathcal{S}\left(\overline{\mathfrak{n}}_{L}\right)$. The normalize Lebesgue measure $d u$ on $\overline{\mathfrak{n}}_{q}^{L}$ given in Section 3 guarantees that $\widehat{\widehat{\phi}}(u)=\phi(-u)$. 
Proposition 4.9. ([27], [17]) Suppose that $X \in \mathfrak{n}_{q}$ with $\nabla_{q}(X) \neq 0$ and $Y^{\prime} \in \overline{\mathfrak{n}}_{n-q}^{\prime}$ with $\bar{\nabla}_{n-q}\left(Y^{\prime}\right) \neq 0$. Then for each $\phi \in \mathcal{S}\left(\overline{\mathfrak{n}}_{q}^{L}\right)$

$$
\int_{\overline{\mathfrak{n}}_{q}^{L}} \int_{\overline{\mathfrak{n}}_{q}^{L}} \phi(v-u) \mathrm{e}^{\pi i\left\langle Y^{\prime}, a d(u)^{2} X\right\rangle} d u d v=\nabla_{q}(X)^{-d(n-q)} \bar{\nabla}_{n-q}\left(Y^{\prime}\right)^{-q d} \int_{\overline{\mathfrak{n}}_{q}^{L}} \phi(u) d u .
$$

Proof. We follow Sections 13 and 14 in [27]. The first step is to see that

$$
q(u) \equiv q_{X, Y^{\prime}}(u) \equiv \mathrm{e}^{\frac{1}{2} i\left\langle Y^{\prime},(a d(u))^{2}(X)\right\rangle}
$$

is a nondegenerate character of the second $\operatorname{degree}^{2}$, that is $q$ satisfies

$$
q(u+v) q(u)^{-1} q(v)^{-1}=\mathrm{e}^{i\left\langle u, \rho_{X, Y^{\prime}}(v)\right\rangle}
$$

for some linear isomorphism $\rho_{X, Y^{\prime}}: \overline{\mathfrak{n}}_{q}^{L} \rightarrow \theta\left(\overline{\mathfrak{n}}_{q}^{L}\right)$.

Since $[u, v]=0, \operatorname{ad}(u) \operatorname{ad}(v)=\operatorname{ad}(v) \operatorname{ad}(u)$. Therefore

$$
\begin{aligned}
& \left\langle Y^{\prime}, \frac{1}{2}(\operatorname{ad}(u) a d(v)+\operatorname{ad}(v) \operatorname{ad}(u))(X)\right\rangle=\left\langle Y^{\prime}, \operatorname{ad}(u) \operatorname{ad}(v) X\right\rangle=-\left\langle\operatorname{ad}(u) Y^{\prime}, \operatorname{ad}(v) X\right\rangle \\
& \quad=-\left\langle a d\left(Y^{\prime}\right) u, \operatorname{ad}(X) v\right\rangle=\left\langle u, \operatorname{ad}\left(Y^{\prime}\right) \operatorname{ad}(X) v\right\rangle .
\end{aligned}
$$

Therefore, taking $\rho_{X, Y^{\prime}}=a d\left(Y^{\prime}\right) \operatorname{ad}(X)$ we see that (4.10) holds. We also need to check that $\rho_{X, Y^{\prime}}$ is a linear isomorphism. By Lemma $3.12 \operatorname{ad}(X): \overline{\mathfrak{n}}_{q}^{L} \rightarrow \widetilde{\mathfrak{n}}$ has determinant $\nabla_{q}(X)^{2 d(n-1)}$. Similarly the determinant of $\operatorname{ad}\left(Y^{\prime}\right): \widetilde{\mathfrak{n}} \rightarrow \theta\left(\overline{\mathfrak{n}}_{q}^{L}\right)$ is $\bar{\nabla}_{n-q}\left(Y^{\prime}\right)^{2 d n}$. Therefore, $q$ is nondegenerate for $X$ and $Y^{\prime}$ satisfying $\nabla_{q}(X) \neq 0$ and $\bar{\nabla}_{n-q}\left(Y^{\prime}\right) \neq 0$. It also follows that the Jacobian term appearing in [27, Cor. 2] is $\nabla_{q}(X)^{-d(n-q)} \bar{\nabla}_{n-q}\left(Y^{\prime}\right)^{-d q}$.

To complete the proof we compute the ' $\gamma$ term' in [27, Cor. 2]. A formula for $\gamma$ is given in [18, Prop. 1-6] in terms of the signature of $\left\langle\cdot, \rho_{X, Y^{\prime}}(\cdot)\right\rangle=\left\langle\cdot, \theta \rho_{X, Y^{\prime}}(\cdot)\right\rangle_{\theta}$. The signature of $\rho_{X, Y^{\prime}}$ is independent of $X, Y^{\prime}$ (with $\nabla_{q}(X) \bar{\nabla}_{n-q}\left(Y^{\prime}\right) \neq 0$ ), so we will assume that

$$
X=\sum_{j=1}^{q} E_{j} \quad \text { and } \quad Y^{\prime}=\sum_{j=q+1}^{n} \theta\left(E_{j}\right) .
$$

Let $\rho=\rho_{X, Y^{\prime}}$. Then we claim that the eigenvalues of $\theta \rho=\theta \rho_{X, Y^{\prime}}$ restricted to $\bar{n}_{q}^{L}$ are \pm 1 , each occurring with the same multiplicity. It follows from this that $\gamma(\rho)=1$.

To see that the claim holds we show that $\theta \rho=\theta \tau$ (with $\tau$ as in (2.7)), then note that the root multiplicities in the 1 and -1 eigenspaces of $\theta \tau$ are all equal (to $d$ ), as observed in [6, Section 1.2].

Both $\theta \rho$ and $\theta \tau$ preserve a-root spaces so it is enough to check that $\rho \theta=\tau \theta$ on root spaces for $-\epsilon_{i}+\epsilon_{k}$, $i \leq q<k$. Let $Z$ be in such a root space, then

$$
\rho(Z)=\left[E_{k}, \theta\left[E_{i}, Z\right]\right]
$$

On the other hand,

$$
\tau \theta(Z)=\operatorname{Ad}\left(\operatorname { e x p } \left(\frac{\pi}{2}\left(E_{i}+\theta\left(E_{i}\right)\right) \exp \left(\frac{\pi}{2}\left(E_{k}+\theta\left(E_{k}\right)\right)\right) \theta(Z)\right.\right.
$$

Now,

$$
\operatorname{Ad}\left(\frac{\pi}{2}\left(E_{k}+\theta\left(E_{k}\right)\right)\right) \theta(Z)=\cos \left(\frac{\pi}{2}\right) \theta(Z)+\sin \left(\frac{\pi}{2}\right)\left[E_{k}, \theta(Z)\right]=\left[E_{k}, Z\right]
$$

${ }^{2}$ See $[27$, pages 145-146] for the definition of a nondegenerate character of the second degree. 
SO

$$
\begin{aligned}
\tau \theta(Z) & =\operatorname{Ad}\left(\exp \left(\frac{\pi}{2}\left(E_{i}+\theta\left(E_{i}\right)\right)\right)\left(\left[E_{k}, \theta(Z)\right]\right)\right. \\
& =\cos \left(\frac{\pi}{2}\right)\left[E_{k}, \theta(Z)\right]+\sin \left(\frac{\pi}{2}\right)\left[\theta\left(E_{i}\right),\left[E_{k}, \theta(Z)\right]\right] \\
& =\left[\theta\left(E_{i}\right),\left[E_{k}, \theta(Z)\right]\right] \\
& =\rho(Z) .
\end{aligned}
$$

This completes the proof.

For $f \in \mathcal{S}(\overline{\mathfrak{n}})$ define $\bar{T}_{f} \in \mathbf{C}^{\infty}\left(\overline{\mathfrak{n}}_{q} \times \overline{\mathfrak{n}}_{n-q}^{\prime}\right)$ by

$$
\bar{T}_{f}\left(Y, Y^{\prime}\right)=\int_{\overline{\mathfrak{n}}_{q}^{L}} f\left(\exp (u)\left(Y+Y^{\prime}\right)\right) d u
$$

and for $h \in \mathcal{S}(\mathfrak{n})$ define $T_{h} \in \mathbf{C}^{\infty}\left(\mathfrak{n}_{q} \times \mathfrak{n}_{n-q}^{\prime}\right)$ by

$$
T_{h}\left(X, X^{\prime}\right)=\int_{\overline{\mathfrak{n}}_{q}^{L}} h\left(\exp (u)\left(X+X^{\prime}\right)\right) d u .
$$

The functions $T_{h}$ and $\bar{T}_{f}$ are not defined everywhere, for example the integral defining $T_{h}(0,0)$ is not convergent (when $h(0,0) \neq 0$ ). The following lemma shows, among other things, that $T_{h}$ and $\bar{T}_{f}$ (for Schwartz functions $h$ and $f$ ) are defined almost everywhere. Before stating the lemma we make several observations.

As noted in Lemma $3.12 \operatorname{ad}(X): \bar{n}_{q}^{L} \rightarrow \widetilde{\mathfrak{n}}$ is invertible when $X \in \mathfrak{n}_{q}$ and $\nabla_{q}(X) \neq 0$, i.e., when $X \in \mathcal{O}_{q}(q)$. This immediately provides us with an estimate in terms of the operator norm of $\operatorname{ad}(X)$ :

$$
\frac{1}{\left\|\operatorname{ad}(X)^{-1}\right\|_{\text {op }}}\|u\| \leq\|\operatorname{ad}(X)(u)\| \leq\|\operatorname{ad}(X)\|_{\text {op }}\|u\|
$$

Since $\|\operatorname{ad}(X)\|_{\text {op }}$ and $\left\|\operatorname{ad}(X)^{-1}\right\|_{\text {op }}$ are continuous on $\mathcal{O}_{q}(q)$ we may conclude that for every compact set $\Omega \subset \mathcal{O}_{q}(q)$ there are constants $C^{\prime}, C^{\prime \prime}>0$ such that

$$
C^{\prime}\|u\| \leq\|\operatorname{ad}(X)(u)\| \leq C^{\prime \prime}\|u\|, \text { for each } X \in \Omega \text {. }
$$

It follows that for each $N \in \mathbf{N}$ there exists a constant $C$ so that

$$
(1+\|\operatorname{ad}(X)(u)\|)^{-N} \leq C(1+\|u\|)^{-N}, \text { for each } X \in \Omega .
$$

Similarly for $Y^{\prime} \in \overline{\mathfrak{n}}_{n-q}^{\prime}$ with $\bar{\nabla}_{n-q}^{\prime}\left(Y^{\prime}\right) \neq 0, \operatorname{ad}\left(Y^{\prime}\right): \overline{\mathfrak{n}}_{q}^{L} \rightarrow \overline{\widetilde{\mathfrak{n}}}$ is invertible and given a compact set $\Omega^{\prime} \subset\left\{Y^{\prime} \in \overline{\mathfrak{n}}_{n-q}^{\prime}: \bar{\nabla}_{n-q}^{\prime}\left(Y^{\prime}\right) \neq 0\right\}$ and an $N \in \mathbf{N}$ there is a constant $C$ so that

$$
\left(1+\left\|\operatorname{ad}\left(Y^{\prime}\right)(u)\right\|\right)^{-N} \leq C(1+\|u\|)^{-N}, \text { for each } Y^{\prime} \in \Omega^{\prime}
$$

Lemma 4.14. Let $h \in \mathcal{S}(\mathfrak{n})$ and $f \in \mathcal{S}(\overline{\mathfrak{n}})$. Then the following statements hold.

(a) The integral defining $T_{h}\left(X, X^{\prime}\right)$ (respectively, $\bar{T}_{f}\left(Y, Y^{\prime}\right)$ ) is finite when $\nabla_{q}(X)$ (respectively, $\bar{\nabla}_{n-q}^{\prime}\left(Y^{\prime}\right)$ ) is nonzero. In particular, $T_{h}$ and $\bar{T}_{f}$ are defined almost everywhere.

(b) For $X \in \mathcal{O}_{q}(q), h\left(X+\operatorname{ad}(u)(X)+X^{\prime}\right)$ is a Schwartz function in the variables $u \in \overline{\mathfrak{n}}_{q}^{L}$ and $X^{\prime} \in \mathfrak{n}_{n-q}^{\prime}$.

(c) Let $\Omega \subset \mathcal{O}_{q}(q)$ be compact and let $N \in \mathbf{N}$. Then there is a constant $C$ so that

$$
T_{h}\left(X, X^{\prime}\right) \leq C\left(1+\left\|X^{\prime}\right\|\right)^{-N}, \text { for all } X \in \Omega .
$$

Furthermore, $T_{h}(X, \cdot) \in \mathcal{S}\left(\mathfrak{n}_{n-q}^{\prime}\right)$ for $\nabla_{q}(X) \neq 0$. 
Proof. (a) Let $\nabla_{q}(X) \neq 0$. Then, for any $N \in \mathbf{N}$ there are constants $C_{1}$ and $C$ so that

$$
\begin{aligned}
\mid h(\exp (u) & \left.\left(X+X^{\prime}\right)\right)|=| h\left(X+\operatorname{ad}(u) X+\left(X^{\prime}+\frac{1}{2} \operatorname{ad}(u)^{2}(X)\right)\right) \mid \\
& \leq C_{1}(1+\|\operatorname{ad}(u) X\|)^{-N}, \text { since } h \text { is Schwartz in each variable, } \\
& \leq C(1+\|u\|)^{-N}, \text { by }(4.11)
\end{aligned}
$$

Choosing $N$ large enough, this is an $L^{1}$ function on $\overline{\mathfrak{n}}_{q}^{L}$. The corresponding statement for $\bar{T}_{f}$ follows from (4.13)

(b) The linear change of coordinates $\operatorname{ad}(X): \overline{\mathfrak{n}}_{q}^{L} \rightarrow \widetilde{\mathfrak{n}}$ sends Schwartz functions to Schwartz functions.

(c) Since $h$ is Schwartz in each of the $\widetilde{\mathfrak{n}}, \mathfrak{n}_{q}$ and $\mathfrak{n}_{n-q}^{\prime}$ variables, for each $M, N \in \mathbf{N}$ there is a constant $C_{1}$ so that

$$
\mid h\left(\exp (u)\left(X+X^{\prime}\right) \mid \leq C_{1}(1+\|\operatorname{ad}(u)(X)\|)^{-M}\left(1+\left\|X^{\prime}+\frac{1}{2} \operatorname{ad}(u)^{2}(X)\right\|\right)^{-N}\right.
$$

Therefore,

$$
\left|T_{h}\left(X, X^{\prime}\right)\right| \leq C_{1}\left(1+\left\|X^{\prime}\right\|\right)^{-N} \int_{\overline{\mathfrak{n}}_{q}^{L}}\left(\frac{1+\left\|X^{\prime}\right\|}{1+\left\|X^{\prime}+\frac{1}{2} \operatorname{ad}(u)^{2}(X)\right\|}\right)^{N}(1+\|\operatorname{ad}(u) X\|)^{-M} d u .
$$

To bound the integrand we use the triangle inequality:

$$
\begin{aligned}
1+\left\|X^{\prime}\right\| & \leq 1+\left\|X^{\prime}+\frac{1}{2} \operatorname{ad}(u)^{2}(X)\right\|+\left\|\frac{1}{2} \operatorname{ad}(u)^{2}(X)\right\| \\
& \leq\left(1+\left\|X^{\prime}+\frac{1}{2} \operatorname{ad}(u)^{2}(X)\right\|\right)\left(1+\left\|\frac{1}{2} \operatorname{ad}(u)^{2}(X)\right\|\right) .
\end{aligned}
$$

Therefore,

$$
\begin{aligned}
\frac{1+\left\|X^{\prime}\right\|}{1+\left\|X^{\prime}+\frac{1}{2} \operatorname{ad}(u)^{2}(X)\right\|} & \leq 1+\frac{1}{2}\|\operatorname{ad}(u)\|_{\text {op }}\|\operatorname{ad}(u) X\| \\
& \leq\left(1+\frac{1}{2}\|\operatorname{ad}(u)\|_{\text {op }}\right)(1+\|\operatorname{ad}(u) X\|) \\
& \leq C_{2}(1+\|u\|)(1+\|\operatorname{ad}(u) X\|), \text { by continuity of ad } \\
& \leq C_{3}(1+\|u\|)^{2}, \text { by }(4.11) .
\end{aligned}
$$

Now choose $M=3 N$, then

$$
\begin{aligned}
T_{h}\left(X, X^{\prime}\right) & \leq C_{3}\left(1+\left\|X^{\prime}\right\|\right)^{-N} \int_{\overline{\mathfrak{n}}_{n-q}^{L}}(1+\|u\|)^{2 N}(1+\|u\|)^{-M} d u \\
& \leq C\left(1+\left\|X^{\prime}\right\|\right)^{-N} .
\end{aligned}
$$

That $T_{h}(X, \cdot)$ is Schwartz follows from the same estimate applied to $\mathfrak{n}_{n-q}^{\prime}$-derivatives of $h$.

Let $\mathcal{F}_{q}$ (respectively, $\mathcal{F}_{n-q}^{\prime}$ ) denote the Fourier transform in the $\mathfrak{n}_{q}$-variable (respectively, the $\mathfrak{n}_{n-q^{-}}$ variable).

Proposition 4.16. ([15], [17]) For $h \in \mathcal{S}(\mathfrak{n}), Y \in \overline{\mathfrak{n}}_{q}$ and $Y^{\prime} \in \overline{\mathfrak{n}}_{n-q}^{\prime}$ with $\bar{\nabla}_{n-q}\left(Y^{\prime}\right) \neq 0$

$$
\bar{T}_{\widehat{h}}\left(Y, Y^{\prime}\right)=\bar{\nabla}_{n-q}\left(Y^{\prime}\right)^{-q d} \mathcal{F}_{q}\left(\nabla_{q}(\cdot)^{d(n-q)} \mathcal{F}_{n-q}\left(T_{h}\right)\right)\left(Y, Y^{\prime}\right) .
$$


Proof. Let $h \in \mathcal{S}(\mathfrak{n})$ and $\bar{\nabla}_{n-q}^{\prime}\left(Y^{\prime}\right) \neq 0$. Then the following integrals converge by part (a) of Lemma 4.14.

$$
\begin{aligned}
\bar{T}_{\widehat{h}}\left(Y, Y^{\prime}\right)=\int_{\overline{\mathfrak{n}}_{q}^{L}} \widehat{h}\left(\exp (u)\left(Y+Y^{\prime}\right)\right) d u \\
=\int_{\overline{\mathfrak{n}}_{q}^{L}}\left(\int_{\mathfrak{n}} h(X) \mathrm{e}^{-2 \pi i\left\langle X, \exp (u)\left(Y+Y^{\prime}\right)\right\rangle} d X\right) d u
\end{aligned}
$$

$$
=\int_{\overline{\mathfrak{n}}_{q}^{L}}\left(\int_{\mathfrak{n}_{q}} \int_{\overline{\mathfrak{n}}_{q}^{L}} \int_{\mathfrak{n}_{n-q}^{\prime}} h\left(\exp (v)\left(X+X^{\prime}\right)\right) \mathrm{e}^{-2 \pi i\left\langle\exp (v)\left(X+X^{\prime}\right), \exp (u)\left(Y+Y^{\prime}\right)\right\rangle} \nabla_{q}(X)^{2 d(n-q)} d X^{\prime} d v d X\right) d u .
$$

We would like to switch the order of integration of the $X$ and $u$ variables. The orthogonality relations $\widetilde{\mathfrak{n}} \perp\left(\mathfrak{n}_{q}+\mathfrak{n}_{n-q}^{\prime}\right), \widetilde{\overline{\mathfrak{n}}} \perp\left(\overline{\mathfrak{n}}_{q}+\overline{\mathfrak{n}}_{n-q}^{\prime}\right), \mathfrak{n}_{n-q}^{\prime} \perp \overline{\mathfrak{n}}_{q}$ and $\overline{\mathfrak{n}}_{n-q}^{\prime} \perp \mathfrak{n}_{q}$ in (4.7) and (4.8) give

$$
\begin{aligned}
\left\langle\exp (v)\left(X+X^{\prime}\right), \exp (u)\left(Y+Y^{\prime}\right)\right\rangle= & \left\langle X^{\prime}+\frac{1}{2} \operatorname{ad}(v)^{2}(X), Y^{\prime}\right\rangle+\langle Y, X\rangle+ \\
& \frac{1}{2}\left\langle X, \operatorname{ad}(u)^{2}\left(Y^{\prime}\right)\right\rangle+\left\langle\operatorname{ad}(u)\left(Y^{\prime}\right), \operatorname{ad}(v)(X)\right\rangle .
\end{aligned}
$$

Moreover, observe that

$$
\begin{aligned}
& \left|\int_{\overline{\mathfrak{n}}_{q}^{L}} \int_{\mathfrak{n}_{n-q}^{\prime}} h\left(\exp (v)\left(X+X^{\prime}\right)\right) \mathrm{e}^{-2 \pi i\left\langle\exp (v)\left(X+X^{\prime}\right), \exp (u)\left(Y+Y^{\prime}\right)\right\rangle} \nabla_{q}(X)^{2 d(n-q)} d X^{\prime} d v\right| \\
& =\nabla_{q}(X)^{2 d(n-q)}\left|\int_{\overline{\mathfrak{n}}_{q}^{L}} \int_{\mathfrak{n}_{n-q}^{\prime}} h\left(X+a d(v) X+X^{\prime}\right) \mathrm{e}^{-2 \pi i\left\langle X^{\prime}, Y^{\prime}\right\rangle} \mathrm{e}^{-2 \pi i\left\langle\operatorname{ad}(u) Y^{\prime}, \operatorname{ad}(v) X\right\rangle} d X^{\prime} d v\right| \\
& \left.=\mid \int_{\tilde{\mathfrak{n}}} \int_{\mathfrak{n}_{n-q}^{\prime}} h\left(X+z+X^{\prime}\right)\right) \mathrm{e}^{-2 \pi i\left\langle Y^{\prime}, X^{\prime}\right\rangle} \mathrm{e}^{-2 \pi i\left\langle a d(u) Y^{\prime}, z\right\rangle} d X^{\prime} \mid d z, \\
& \quad \text { by applying the change of variables } z=-\operatorname{ad}(X)(v) \text { and Lemma 3.14, } \\
& =\left|\int_{\tilde{\mathfrak{n}}} \mathcal{F}_{n-q}(h(X+z+\cdot))\left(Y^{\prime}\right) \mathrm{e}^{-2 \pi i\left\langle a d(u) Y^{\prime}, z\right\rangle} d z\right| \\
& =\mid\left(\mathcal{F}_{z} \mathcal{F}_{n-q} h(X+\cdot+\cdot)\left(\operatorname{ad}(u) Y^{\prime}, Y^{\prime}\right) \mid .\right.
\end{aligned}
$$

Where $\mathcal{F}_{z}$ is the Fourier transform in the $\widetilde{\mathfrak{n}}$ variable. Since $h$ is Schwartz in each of the three variables so are the Fourier transforms. Therefore we may bound the above expression by

$$
C^{\prime}(1+\|X\|)^{-N}\left(1+\left\|\operatorname{ad}(u) Y^{\prime}\right\|\right)^{-N}
$$

which is bounded by

$$
C(1+\|X\|)^{-N}(1+\|u\|)^{-N}
$$

for some $C$ (depending on $Y^{\prime}$ ) by (4.12). Now Fubini's Theorem allows us rewrite the expression (4.17):

$$
\begin{aligned}
\bar{T}_{\widehat{h}} & \left(Y, Y^{\prime}\right)= \\
& =\int_{\mathfrak{n}_{q}} \nabla_{q}(X)^{2 d(n-q)}\left(\int_{\overline{\mathfrak{n}}_{q}^{L}} \int_{\overline{\mathfrak{n}}_{q}^{L}}\left\{\int_{\mathfrak{n}_{n-q}^{\prime}} h\left(\exp (v-u)\left(X+X^{\prime}\right)\right) \mathrm{e}^{2 \pi i\left\langle X+X^{\prime}, \exp (u)\left(Y+Y^{\prime}\right)\right\rangle} d X^{\prime}\right\} d v d u\right) d X= \\
& =\int_{\mathfrak{n}_{q}} \nabla_{q}(X)^{2 d(n-q)} \mathrm{e}^{2 \pi i\langle Y, X\rangle}\left(\int_{\overline{\mathfrak{n}}_{q}^{L}} \int_{\overline{\mathfrak{n}}_{q}^{L}}\left\{\int_{\mathfrak{n}_{n-q}^{\prime}} h\left(\exp (v-u)\left(X+X^{\prime}\right)\right) \mathrm{e}^{2 \pi i\left\langle X^{\prime}, Y^{\prime}\right\rangle} \mathrm{e}^{2 \pi i\left\langle X, \frac{1}{2} a d(u)^{2} Y^{\prime}\right\rangle} d X^{\prime}\right\} d v d u\right) d X= \\
& =\int_{\mathfrak{n}_{q}} \nabla_{q}(X)^{2 d(n-q)} \mathrm{e}^{2 \pi i\langle Y, X\rangle}\left(\int_{\overline{\mathfrak{n}}_{q}^{L}} \int_{\overline{\mathfrak{n}}_{q}^{L}} \phi_{X, Y^{\prime}}(v-u) \mathrm{e}^{2 \pi i\left\langle X, \frac{1}{2} a d(u)^{2} Y^{\prime}\right\rangle} d v d u\right) d X,
\end{aligned}
$$

where

$$
\phi_{X, Y^{\prime}}(u)=\int_{\mathfrak{n}_{n-q}^{\prime}} h\left(\exp (u)\left(X+X^{\prime}\right) \mathrm{e}^{2 \pi i\left\langle Y^{\prime}, X^{\prime}\right\rangle} d X^{\prime}\right.
$$


In order to apply Weil's formula (Prop. (4.9)) we need to know that for $X \in \mathcal{O}_{q}(q), \phi_{X, Y^{\prime}} \in \mathcal{S}\left(\overline{\mathfrak{n}}_{q}^{L}\right)$. To check this we apply (4.11):

$$
\begin{aligned}
\left|\phi_{X, Y^{\prime}}(u)\right| & =\left|\int_{\mathfrak{n}_{n-q}^{\prime}} h\left(X+\operatorname{ad}(u) X+\left(X^{\prime}+\frac{1}{2} \operatorname{ad}(u)^{2}(X)\right)\right) \mathrm{e}^{2 \pi i\left\langle Y^{\prime}, X^{\prime}\right\rangle} d X^{\prime}\right| \\
= & \left|\int_{\mathfrak{n}_{n-q}^{\prime}} h\left(X+\operatorname{ad}(u) X+X^{\prime}\right) \mathrm{e}^{2 \pi i\left\langle Y^{\prime}, X^{\prime}-\frac{1}{2} \operatorname{ad}(u)^{2}(X)\right\rangle} d X^{\prime}\right| \\
\leq & \int_{\mathfrak{n}_{n-q}^{\prime}}\left|h\left(X+\operatorname{ad}(u) X+X^{\prime}\right)\right| d X^{\prime} \\
\leq & C^{\prime} \int_{\mathfrak{n}_{n-q}^{\prime}}\left(1+\left\|X^{\prime}\right\|\right)^{-M}(1+\|\operatorname{ad}(u) X\|)^{-N} d X^{\prime} \\
\leq & C(1+\|u\|)^{-N}, \text { by }(4.12) .
\end{aligned}
$$

Derivatives in the $\overline{\mathfrak{n}}_{q}^{L}$ directions also satisfy this type of estimate since these derivatives are just derivatives of $h$ times polynomials in $X$ and $u$.

Now we may apply Prop. 4.9. Suppose $\bar{\nabla}_{n-q}^{\prime}\left(Y^{\prime}\right) \neq 0$.

$$
\begin{aligned}
\bar{T}_{\widehat{h}}\left(Y, Y^{\prime}\right) & =\bar{\nabla}_{n-q}\left(Y^{\prime}\right)^{-d q} \int_{\mathfrak{n}_{q}} \nabla_{q}(X)^{d(n-q)} \mathrm{e}^{2 \pi i\langle Y, X\rangle}\left(\int_{\overline{\mathfrak{n}}_{q}^{L}} \int_{\mathfrak{n}_{n-q}^{\prime}} h\left(\exp (u)\left(X+X^{\prime}\right)\right) \mathrm{e}^{2 \pi i\left\langle Y^{\prime}, X^{\prime}\right\rangle} d X^{\prime} d u\right) d X \\
& =\bar{\nabla}_{n-q}\left(Y^{\prime}\right)^{-d q} \int_{\mathfrak{n}_{q}} \nabla_{q}(X)^{d(n-q)} \mathrm{e}^{2 \pi i\langle Y, X\rangle}\left(\int_{\mathfrak{n}_{n-q}^{\prime}} \int_{\overline{\mathfrak{n}}_{q}^{L}} h\left(\exp (u)\left(X+X^{\prime}\right)\right) \mathrm{e}^{2 \pi i\left\langle Y^{\prime}, X^{\prime}\right\rangle} d u d X^{\prime}\right) d X,
\end{aligned}
$$

by Fubini's Theorem (using part (b) of Lemma 4.14),

$$
=\bar{\nabla}_{n-q}\left(Y^{\prime}\right)^{-q d} \mathcal{F}_{q}\left(\nabla_{q}(\cdot)^{d(n-q)} \mathcal{F}_{n-q}\left(T_{h}\right)\right)\left(Y, Y^{\prime}\right)
$$

Now fix some $q$ satisfying $1 \leq q \leq n-1$ and recall that $\mathcal{U}_{q}=\left\{X \in \mathfrak{n}: \nabla_{q}\left(\pi_{q}(X)\right) \neq 0\right\}$, an open set in $\mathfrak{n}$. Let $C_{c}^{\infty}(\mathfrak{n})$ be the space of smooth functions on $\mathfrak{n}$ having compact support. Suppose $h \in C_{c}^{\infty}$ and $\operatorname{supp}(h) \subset \mathcal{U}_{q}$. Then $h\left(\exp (u)\left(X+X^{\prime}\right)=h\left(X+\operatorname{ad}(u) X+\left(X^{\prime}+\frac{1}{2} \operatorname{ad}(u)^{2} X\right)\right)\right.$ is smooth and has compact support contained in $\mathcal{O}_{q}(q) \times \overline{\mathfrak{n}}_{q}^{L} \times \mathfrak{n}_{n-q}^{\prime}$, by Lemma 3.14. This implies the following lemma.

Lemma 4.18. If $h \in C_{c}^{\infty}$ and $\operatorname{supp}(h) \subset \mathcal{U}_{q}$ then $T_{h} \in C_{c}^{\infty}\left(\mathfrak{n}_{q} \times \mathfrak{n}_{n-q}^{\prime}\right)$. The support of $T_{h}$ is contained in $\mathcal{O}_{q}(q) \times \mathfrak{n}_{n-q}^{\prime}$.

Recall that $\mathcal{O}_{n}=\{X \in \mathfrak{n}: \nabla(X) \neq 0\}$.

Lemma 4.19. Let $1 \leq q \leq n-1$ and suppose that the support of $h$ is compact and contained in $\mathcal{U}_{q} \cap \mathcal{O}_{n}$. Then the following hold.

(1) $Z_{n-q}\left(T_{h}(X, \cdot), s\right)$ is a smooth function of compact support in the $X$-variable.

(2) $Z_{n}(h, s)=Z_{q}\left(\nabla_{q}^{2 d(n-q)} Z_{n-q}\left(T_{h}, s\right), s\right)$, as meromorphic functions of $s$.

Proof. (1) Note that if $\exp (u)\left(X+X^{\prime}\right) \in \mathcal{U}_{q} \cap \mathcal{O}_{n}$ then $\nabla_{n-q}\left(X^{\prime}\right) \neq 0$. Therefore

$$
Z_{n-q}\left(T_{h}(X, \cdot), s\right)=\int_{\mathfrak{n}_{n-q}^{\prime}} T_{h}\left(X, X^{\prime}\right) \nabla_{n-q}\left(X^{\prime}\right)^{s} d X^{\prime}
$$

for all $X \in \mathfrak{n}_{q}$ and all $s \in \mathbf{C}$. By differentiating inside the integral we see that $Z_{n-q}\left(T_{h}(X, \cdot), s\right)$ is smooth. By Lemma (4.18), $Z_{n-q}\left(T_{h}(X, \cdot), s\right)$ is of compact support. 
(2) All integrals below converge for all $s \in \mathbf{C}$.

$$
\begin{aligned}
Z_{n}(h, s)= & \int_{\mathfrak{n}} h(X) \nabla(X)^{s} d X \\
= & \int_{\mathfrak{n}_{q}} \int_{\mathfrak{n}_{n-q}^{\prime}} \int_{\overline{\mathfrak{n}}_{q}^{L}} h\left(\exp (u)\left(X+X^{\prime}\right)\right) \nabla\left(\exp (u)\left(X+X^{\prime}\right)\right)^{s} \nabla(X)^{2 d(n-q)} d u d X^{\prime} d X \\
& \quad \text { by Lemma } 3.13, \\
= & \int_{\mathfrak{n}_{q}} \int_{\mathfrak{n}_{n-q}^{\prime}} T_{h}\left(X, X^{\prime}\right) \nabla_{q}(X)^{s+2 d(n-q)} \nabla_{n-q}\left(X^{\prime}\right)^{s} d X d X^{\prime} \\
= & \int_{\mathfrak{n}_{q}} Z_{n-q}\left(T_{h}(X, \cdot), s\right) \nabla_{q}(X)^{s+2 d(n-q)} d X \\
= & Z_{q}\left(\nabla(\cdot)^{2 d(n-q)} Z_{n-q}\left(T_{h}, s\right), s\right) .
\end{aligned}
$$

The last line makes sense by (1).

Remark 4.21. The following refinement of the above lemma holds and will be used in Section 5. Suppose that the hypothesis of the lemma is weakened to $\operatorname{supp}(h) \subset \mathcal{U}_{q}$. Then for $\operatorname{Re}(s) \gg 0$

$$
Z_{n}(h, s)=\int_{\mathfrak{n}_{q}} Z_{n-q}\left(T_{h}(X, \cdot), s\right) \nabla_{q}(X)^{s+2 d(n-q)} d X
$$

as in (4.20). By formula (B.1) and meromorphic continuation $Z_{n-q}\left(T_{h}(X, \cdot), s\right)$ has compact support for $s \notin S$ (= set of potential poles). Furthermore, formula (B.1) shows that $Z_{n-q}\left(T_{h}(X, \cdot), s\right)$ is defined by an integral and is smooth in $X$. Therefore, the integrand in (4.22) is smooth with compact support away from $\nabla_{q}=0$. Thus, the right hand side of $(4.22)$ is

$$
Z_{q}\left(\nabla(\cdot)^{2 d(n-q)} Z_{n-q}\left(T_{h}, s\right), s\right)
$$

for all $s$. Therefore, by meromorphic continuation statement (2) of the lemma holds as meromorphic functions.

We now give the proof of Theorem 4.4. By [22][Thm. 1] there is a functional equation of the form

$$
\bar{Z}\left(\widehat{h}, s-\frac{m}{n}\right)=\beta_{n}(s) Z(h,-s), \quad h \in \mathcal{S}(\mathfrak{n})
$$

for some meromorphic function $\beta_{n}$. Therefore, to prove the explicit functional equation stated in Prop. 4.4 we need to show

$$
\beta_{n}(s)=\pi^{-n s+\frac{m}{2}} \frac{\Gamma_{n}(s)}{\Gamma_{n}\left(-s+\frac{m}{n}\right)}
$$

We proceed by induction on the rank of $\mathfrak{n}$. For $\operatorname{rank}(\mathfrak{n})=1$ the the formula (4.23) is contained in Prop. C.2.

Assume (4.23) holds for all $\mathfrak{n}$ of rank less than $n$. It suffices to assume that $h \in C_{c}^{\infty}(\mathfrak{n})$ and $\operatorname{supp}(h) \subset$ $\mathcal{U}_{q} \cap \mathcal{O}_{n}$. We may take $q=1$. Assume that $\operatorname{Re}(s) \gg 0$, the integrals below converge. 


$$
\begin{aligned}
Z\left(\widehat{h}, s-\frac{m}{n}\right)= & \int_{\overline{\mathfrak{n}}^{\prime}} \widehat{h}(Y) \bar{\nabla}(Y)^{s-\frac{m}{n}} d Y \\
= & \int_{\overline{\mathfrak{n}}_{1}} \int_{\overline{\mathfrak{n}}_{n-1}^{\prime}} \int_{\overline{\mathfrak{n}}_{1}^{L}} \widehat{h}\left(\exp (u)\left(Y+Y^{\prime}\right)\right) \bar{\nabla}\left(\exp (u)\left(Y+Y^{\prime}\right)\right)^{s-\frac{m}{n}} \bar{\nabla}_{n-1}\left(Y^{\prime}\right)^{2 d} d u d Y^{\prime} d Y \\
& \quad \text { y Lemma } 3.13, \\
= & \int_{\overline{\mathfrak{n}}_{1}} \int_{\overline{\mathfrak{n}}_{n-1}^{\prime}} \bar{T}_{\widehat{h}}\left(Y+Y^{\prime}\right) \bar{\nabla}_{1}(Y)^{s-\frac{m}{n}} \bar{\nabla}_{n-1}\left(Y^{\prime}\right)^{s-\frac{m}{n}+2 d} d Y^{\prime} d Y \\
= & \int_{\overline{\mathfrak{n}}_{1}} \int_{\overline{\mathfrak{n}}_{n-1}^{\prime}} \mathcal{F}_{1}\left(\nabla(\cdot)^{d(n-1)} \mathcal{F}_{n-1}\left(T_{h}\right)\right) \bar{\nabla}(Y)_{1}^{s-\frac{m}{n}} \bar{\nabla}_{n-1}\left(Y^{\prime}\right)^{s-\frac{m}{n}+d} d Y^{\prime} d Y, \\
& \quad \text { by Prop. } 4.16, \\
= & \bar{Z}_{1}\left(\mathcal{F}_{1}\left(\nabla(\cdot)^{d(n-1)} \bar{Z}_{n-1}\left(\mathcal{F}_{n-1}\left(T_{h}\right), s-\frac{m_{n-1}}{n-1}\right), s-\frac{m}{n}\right),\right. \\
& \quad \text { since the order of integration over } \overline{\mathfrak{n}}_{n-1}^{\prime} \text { and the integral defining } \mathcal{F}_{1} \\
& \quad \text { may be interchanged by Lemma } 4.18, \\
= & \beta_{1}(s) \beta_{n-1}(s-(n-1) d) Z_{1}\left(\nabla_{1}(\cdot)^{d(n-1)} Z_{n-1}\left(T_{h},-s\right),-\left(s-\frac{m}{n}+e+1\right)\right) \\
= & \pi^{-n s+\frac{m}{2}} \frac{\Gamma_{n}(s)}{\Gamma_{n}\left(-s+\frac{m}{n}\right)} Z_{1}\left(Z_{n-1}\left(T_{h},-s\right),-s+2 d(n-1)\right), \text { by induction and }(4.24) \\
= & \pi^{-n s+\frac{m}{2}} \frac{\Gamma_{n}(s)}{\Gamma_{n}\left(-s+\frac{m}{n}\right)} \mathbf{Z}_{n}(h,-s), \text { by Lemma } 4.19(\text { with } q=1) .
\end{aligned}
$$

We have used the following fact, which is an easy calculation.

$$
\frac{\pi^{\frac{1}{2}(-s+(e+1))} \Gamma_{1}(s)}{\pi^{\frac{s}{2}} \Gamma_{1}(-s+(e+1))} \frac{\pi^{\frac{n-1}{2}\left(-(s-d)+\frac{m_{n-1}}{n-1}\right)} \Gamma_{n-1}(s-d)}{\pi^{\frac{n-1}{2}(s-d)} \Gamma_{n-1}\left(-(s-d)+\frac{\left.m_{n-1}\right)}{n-1}\right)}=\frac{\pi^{\frac{n}{2}\left(-s+\frac{m}{n}\right)} \Gamma_{n}(s)}{\pi^{\frac{n s}{2}} \Gamma_{n}\left(-s+\frac{m}{n}\right)} .
$$

The formula for $\beta_{n}(s)$ now follows by meromorphic continuation and Theorem 4.4 is proved.

A few important consequences of the Theorem (and proof) follow.

Corollary 4.25. $b(s)=\prod_{j=0}^{n-1}(s+j d)(s+(e-1)+j d)$. Therefore the set of potential poles of $Z(h, s)$ is $S=$ $S^{\prime} \cup S^{\prime \prime}=\left\{-j d+2 l: l \in \mathbf{Z}_{+}\right\} \cup\left\{j d-\frac{m}{n}+2 l: l \in \mathbf{Z}_{+}\right\}$.

Proof. Use $\bar{P}\left(\partial_{Y}\right) f$ in place of $f$ and $-s$ in place of $s$ in the functional equation. The

$$
\begin{aligned}
\mathbf{Z}\left(\widehat{P\left(\partial_{Y}\right) f},\right. & \left.-s-\frac{m}{n}\right)=\mathbf{Z}\left((2 \pi i)^{2 n} P(X) \widehat{f},-s-\frac{m}{n}\right) \\
& =(-1)^{n}(2 \pi)^{2 n} \mathbf{Z}\left(\widehat{f},-s+2-\frac{m}{n}\right) \\
& =(-1)^{n} 2^{2 n} \pi^{\frac{m}{2}+n(s)} \frac{\Gamma_{n}(-s+2)}{\Gamma_{n}\left(s-2+\frac{m}{n}\right)} \overline{\mathbf{Z}}(f, s-2) .
\end{aligned}
$$

Also,

$$
\begin{gathered}
\mathbf{Z}\left(\widehat{\overline{P\left(\partial_{Y}\right)} f},-s-\frac{m}{n}\right)=\pi^{\frac{m}{2}+n s} \frac{\Gamma_{n}(-s)}{\Gamma_{n}\left(s+\frac{m}{n}\right)} \overline{\mathbf{Z}}\left(\bar{P}\left(\partial_{Y}\right) f, s\right) \\
=\pi^{\frac{m}{2}+n s} \frac{\Gamma_{n}(-s)}{\Gamma_{n}\left(s+\frac{m}{n}\right)} b(s) \overline{\mathbf{Z}}(f, s-2) .
\end{gathered}
$$

Therefore,

$$
b(s)=2^{2 n} \frac{\Gamma_{n}\left(s+\frac{m}{n}\right) \Gamma_{n}(-s+2)}{\Gamma_{n}\left(s-2+\frac{m}{n}\right) \Gamma_{n}(-s)} .
$$


Now the corollary follows from the formula

$$
\frac{\Gamma_{n}(u+2)}{\Gamma_{n}(u)}=\prod_{j=0}^{n-1} \frac{u-d j}{2}
$$

Corollary 4.26. $\frac{1}{\Gamma_{n}\left(s+\frac{m}{n}\right)} Z(h, s)$ is an analytic function of $s$ on the complement of $[-(n-1) d,-(e+1)] \cap S$. Proof. If $\operatorname{Re}(s)>-(e+1)$ then $Z(h, s)$ is defined by an integral and is therefore analytic in this range. Applying the functional equation we get analyticity for $\operatorname{Re}(s)<-(n-1) d$.

\section{Special Values of the Zeta Distributions}

In this section we prove Theorem 5.12 which states that the normalized zeta distribution (5.13) is an entire function of $s$ and at certain special values of $s$ is a quasi-invariant measure on an $L$-orbit in $\mathfrak{n}$.

Recall from (3.7) that a quasi-invariant measure on the $L$-orbit $\mathcal{O}_{q}$ satisfies

$$
\int_{\mathcal{O}_{q}}(\ell \cdot h)(X) d \nu_{q}(X)=\chi(\ell)^{q} \int_{\mathcal{O}_{q}} h(X) d \nu_{q}(X)
$$

where $(\ell \cdot h)(X)=h\left(\ell^{-1} \cdot X\right)$. Zeta distributions satisfy the similar homogeneity property

$$
Z(\ell \cdot h, s)=\chi(\ell)^{\frac{1}{d}\left(s+\frac{m}{n}\right)} Z(h, s) .
$$

Lemma 5.2. For $h \in \mathcal{S}(\mathfrak{n})$ the following hold.

(1) $\left.\frac{1}{\Gamma_{n}\left(s+\frac{m}{n}\right)} Z(h, s)\right|_{s=-\frac{m}{n}}=\frac{\pi^{\frac{m}{2}}}{\Gamma_{n}\left(\frac{m}{n}\right)} h(0)$.

(2) Let $x_{1}, \ldots, x_{k}$ be a-root vectors in $\mathfrak{n}$. Write $H=\sum H_{i}$ (with $H_{i}$ as in Section 2) and $a_{t}=\exp (t H)$. Then

$$
\left(x_{1} \cdots x_{k} \cdot \delta_{0}\right)\left(a_{t} \cdot h\right)=\chi\left(a_{t}\right)^{-\frac{k}{d n}}\left(x_{1} \cdots x_{k} \cdot\right) \delta_{0}(h)
$$

Proof. (1) This follows immediately from the functional equation (4.5), Fourier inversion and the fact that $\overline{\mathbf{Z}}(\widehat{h}, s)$ is defined at $s=0$ by an integral.

$$
\begin{gathered}
\left.\frac{1}{\Gamma_{n}\left(s+\frac{m}{n}\right)} Z(h, s)\right|_{s=-\frac{m}{n}}=\frac{\pi^{\frac{m}{2}}}{\Gamma_{n}\left(\frac{m}{n}\right)} \overline{\mathbf{Z}}(\widehat{h}, 0) \\
=\frac{\pi^{\frac{m}{2}}}{\Gamma_{n}\left(\frac{m}{n}\right)} \int_{\overline{\mathfrak{n}}} \widehat{h}(Y) d Y \\
=\frac{\pi^{\frac{m}{2}}}{\Gamma_{n}\left(\frac{m}{n}\right)} h(0) .
\end{gathered}
$$

(2) Observe that $\operatorname{ad}(H)\left(x_{j}\right)=2 x_{j}$ for each root vector $x_{j}$. Also note that for any tempered distribution $T$, $\left(x_{j} \cdot T\right)(\ell \cdot h)=\left(\left(\operatorname{Ad}\left(\ell^{-1}\right) x_{j}\right) \cdot T\right)(h)$, for any $\ell \in L$. Thus,

$$
\begin{aligned}
\left(x_{1} \cdots x_{k} \cdot \delta_{0}\right)\left(a_{t} \cdot h\right) & =\left(\operatorname{Ad}\left(a_{t}^{-1}\right) x_{1} \cdots \operatorname{Ad}\left(a_{t}^{-1}\right) x_{k} \cdot \delta_{0}\right)(h) \\
& =\mathrm{e}^{-2 k t}\left(x_{1} \cdots x_{k} \cdot \delta_{0}\right)(h) \\
& =\chi\left(a_{t}\right)^{-\frac{k}{d n}}\left(x_{1} \cdots x_{k} \cdot \delta_{0}\right)(h) .
\end{aligned}
$$


At this point we normalize the quasi invariant measures on $\mathcal{O}_{q} \subset \mathfrak{n}$. Recall that the Lebesgue measures on $\mathfrak{n}$ and $\overline{\mathfrak{n}}$, and subspaces $\mathfrak{n}_{q}, \overline{\mathfrak{n}}_{q}^{L}$, etc., are normalized in Section 3 in terms of the inner product $\langle,\rangle_{\theta}$. Let

$$
C_{q}^{n}=\frac{\pi^{\frac{m_{n-q}}{2}}}{\Gamma_{q}(d q) \Gamma_{n-q}\left(\frac{m_{n-q}}{n-q}\right)} .
$$

We take $\nu_{q}$ to be normalized so that

$$
\begin{aligned}
\nu_{0} & =C_{0}^{n} \delta_{0} \\
\int_{\mathcal{O}_{q}} F(X) d \nu_{q}(X) & =C_{q}^{n} \int_{\overline{\mathfrak{n}}_{q}^{L}} \int_{\mathfrak{n}_{q}} F(\exp (u) \cdot X) \nabla_{q}(X)^{n d-\frac{m_{q}}{q}} d X d u, \text { for } q=1,2, \ldots, n-1 .
\end{aligned}
$$

Proposition 5.5. Let $\nu_{q}$ be the quasi-invariant measure on $\mathcal{O}_{q}$, for $q=0,1, \ldots, n-1$. For $h$ a smooth function of compact support

$$
\left.\frac{1}{\Gamma_{n}\left(s+\frac{m}{n}\right)} Z(h, s)\right|_{s=-\frac{m}{n}+q d}=\int_{\mathcal{O}_{q}} f d \nu_{q} .
$$

Proof. Our proof is based on Remark 4.21 and the formula for the quasi-invariant measure given in Cor. 3.11. We proceed by induction on $n$, the rank of $\mathfrak{n}$. If $n=1$ the statement is well-known (and follows from Prop. C.2 or Lemma $5.2(2))$.

Suppose first that $h \in \mathcal{S}(\mathfrak{n})$ and has compact support in

$$
\mathcal{U}_{1}=\left\{X \in \mathfrak{n}: \nabla_{1}\left(\pi_{1}(X)\right) \neq 0\right\}
$$

where $\pi_{1}: \mathfrak{n} \rightarrow \mathfrak{n}_{1}$ is the orthogonal projection (with respect to $\langle,\rangle_{\theta}$ ). Then Remark (4.21) applies (with $q=1)$ to give

$$
\begin{gathered}
\left.\frac{1}{\Gamma_{n}\left(s+\frac{m}{n}\right)} Z(h, s)\right|_{-\frac{m}{n}+q d}=\left.\frac{1}{\Gamma_{1}\left(s+\frac{m}{n}\right)} \int_{\mathfrak{n}_{1}} \frac{1}{\Gamma_{n-1}\left(s+\frac{m_{n-1}}{n-1}\right)} Z_{n-1}\left(T_{h}(X, \cdot), s\right) \nabla_{1}(X)^{2 d(n-1)+s} d X\right|_{-\frac{m}{n}+q d} \\
=\frac{1}{\Gamma_{1}(d q)} \int_{\mathfrak{n}_{1}} \int_{\mathcal{O}_{q-1}(n-1)} T_{h}\left(X, X^{\prime}\right) d \nu_{q-1}^{n-1}\left(X^{\prime}\right) \nabla_{1}(X)^{2 d(n-1)-\frac{m}{n}+q d} d X .
\end{gathered}
$$

The last equality follows from the inductive hypothesis (since $\left.-\frac{m}{n}+q d=-\frac{m_{n-1}}{n-1}+(q-1) d\right)$. Here, $\mathcal{O}_{q-1}(n-1)$ is the orbit of $L_{n-1}$ in $\mathfrak{n}_{n-1}^{\prime}$ through $E_{2}+\cdots E_{q}$ and $\nu_{q-1}^{n-1}$ is the quasi-invariant measure on this orbit.

In order to apply Cor. 3.13 we will use the following temporary notation. (This notation is consistent with (4.6) with $\mathfrak{n}$ replaced by $\mathfrak{n}_{q}$ or $\mathfrak{n}_{n-1}$.)

$$
\begin{aligned}
& \mathfrak{n}_{n-1, q-1}^{\prime}=\mathfrak{n}_{q, q-1}^{\prime}=\sum \mathfrak{g}^{\left(\epsilon_{i}+\epsilon_{j}\right)}, \quad 2 \leq i, j \leq q \\
& \overline{\mathfrak{n}}_{n-1, q-1}^{\prime L}=\sum \mathfrak{g}^{-\left(\epsilon_{i}-\epsilon_{j}\right)}, \quad 2 \leq i \leq q<j \leq n \\
& \overline{\mathfrak{n}}_{q, 1}^{L}=\sum \mathfrak{g}^{-\left(\epsilon_{1}-\epsilon_{j}\right)}, \quad 2 \leq j \leq q .
\end{aligned}
$$

Note that

$$
\begin{aligned}
& \overline{\mathfrak{n}}_{1}^{L}=\left(\overline{\mathfrak{n}}_{1}^{L} \cap \overline{\mathfrak{n}}_{q}^{L}\right) \oplus \overline{\mathfrak{n}}_{q, 1}^{L} \\
& \overline{\mathfrak{n}}_{q}^{L}=\left(\overline{\mathfrak{n}}_{1}^{L} \cap \overline{\mathfrak{n}}_{q}^{L}\right) \oplus \overline{\mathfrak{n}}_{n-1, q-1}^{L} .
\end{aligned}
$$

The integration formula of Cor. 3.13 applied to $\mathfrak{n}_{q}$ is

$$
\int_{\mathfrak{n}_{q}} g(X) d X=\int_{\mathfrak{n}_{1}} \int_{\overline{\mathfrak{n}}_{q, 1}^{L}} \int_{\mathfrak{n}_{q, q-1}^{\prime}} g\left(\exp \left(u_{1}\right)\left(X_{1}+X_{2}\right)\right) \nabla_{1}\left(X_{1}\right)^{2 d(q-1)} d X_{1} d u_{1} d X_{2} .
$$

One more bit of notation used below is that $\nabla_{n-1, q-1}$ is the $\nabla$ function for $\mathfrak{n}_{n-1, q-1}^{\prime}=\mathfrak{n}_{q, q-1}^{\prime}$. 
Continuing with (5.6) we have

$$
\begin{aligned}
\left.\frac{1}{\Gamma_{n}\left(s+\frac{m}{n}\right)} Z(h, s)\right|_{-\frac{m}{n}+q d} & \frac{1}{\Gamma_{1}(q d)} \int_{\mathfrak{n}_{1}} C_{q-1}^{n-1} \int_{\overline{\mathfrak{n}}_{n-1, q-1}^{L}} \int_{\mathfrak{n}_{n-1, q-1}^{\prime}} T_{h}\left(X, \exp (v) X^{\prime}\right) \nabla_{n-1, q-1}\left(X^{\prime}\right)^{d(n-1)-\frac{m_{q-1}}{q-1}} \\
& \nabla_{1}(X)^{2 d(n-1)-\frac{m}{n}+q d} d X^{\prime} d v d X \text { by Cor. } 3.11 \\
= & C_{q}^{n} \int_{\overline{\mathfrak{n}}_{1}^{L} \cap \overline{\mathfrak{n}}_{q}^{L}} \int_{\overline{\mathfrak{n}}_{n-1, q-1}^{\prime L}}\left(\int_{\overline{\mathfrak{n}}_{q, 1}^{L}} \int_{\mathfrak{n}_{1}} \int_{\mathfrak{n}_{q, q-1}^{\prime}} h\left(\exp \left(u_{1}+u_{2}\right)\left(X+\exp (v) X^{\prime}\right)\right)\right. \\
= & C_{q}^{n} \int_{\overline{\mathfrak{n}}_{q}^{L}} \int_{\mathfrak{n}_{q}} h(\exp (u) X) \nabla_{q}(X)^{n d-\frac{m_{q}}{q}} d X d u, \text { see below for verification } \\
= & \int_{\mathcal{O}_{q}} h d \nu_{q} .
\end{aligned}
$$

To verify (5.10) note that for $u_{1} \in \overline{\mathfrak{n}}_{q, 1}^{L}, u_{2} \in \overline{\mathfrak{n}}_{1}^{L} \cap \overline{\mathfrak{n}}_{q}^{L}, v \in \overline{\mathfrak{n}}_{n-1, q-1}^{L}$ and $X \in \mathfrak{n}_{1}$

(i) $\operatorname{Ad}\left(\exp (v)^{-1}\right) u_{1}=u_{1}-\left[v, u_{1}\right]$, and $\left[v, u_{1}\right] \in \overline{\mathfrak{n}}_{1}^{L} \cap \overline{\mathfrak{n}}_{q}^{L}$,

(ii) $u_{1}$ and $\left[v, u_{1}\right]$ commute,

(iii) $u_{1}$ and $u_{2}$ commute,

(iv) $u_{2},\left[v, u_{1}\right]$ and $v$ mutually commute and

(v) $v$ commutes with $X$.

Therefore,

$$
\begin{gathered}
\exp \left(u_{1}+u_{2}\right)\left(X+\exp (v) X^{\prime}\right)=\exp \left(u_{2}\right) \exp (v) \exp \left(\operatorname{Ad}\left(\exp (v)^{-1}\right) u_{1}\right)\left(X+X^{\prime}\right) \\
=\exp (v) \exp \left(u_{2}\right) \exp \left(u_{1}-\left[v, u_{1}\right]\right)\left(X+X^{\prime}\right) \\
=\exp (v) \exp \left(u_{2}-\left[v, u_{1}\right]\right) \exp \left(u_{1}\right)\left(X+X^{\prime}\right) .
\end{gathered}
$$

By translation invariance of $d u_{2}$ the line before (5.10) has integrand $h\left(\exp \left(v+u_{2}\right) \exp \left(u_{1}\right)\left(X+X^{\prime}\right)\right)$. Now, line (5.10) follows from (5.8) and (5.9).

We have proved that

$$
\left.\frac{1}{\Gamma_{n}\left(s+\frac{m}{n}\right)} Z(h, s)\right|_{s=-\frac{m}{n}+q d}=\int_{\mathcal{O}_{q}} h(X) d \nu_{q}(X)
$$

for smooth functions $h$ having compact support in $\mathcal{U}_{1}$. Now let $h$ have compact support in $\mathfrak{n} \backslash\{0\}$. Since each positive dimensional $L$-orbit in $\mathfrak{n}$ meets $\mathcal{U}_{1}, \mathfrak{n} \backslash\{0\}=\cup_{\ell \in L} \ell \cdot \mathcal{U}_{1}$. Therefore, by compactness of the support of $h$ we may find $\ell_{1}, \ldots, \ell_{k} \in L$ so that $\operatorname{supp}(h) \subset \cup_{j=1}^{k} \ell_{j} \cdot \mathcal{U}_{1}$. 
Choose a smooth partition of unity $\left\{\phi_{j}\right\}$ subordinate to $\left\{\ell_{j} \cdot \mathcal{U}_{1}\right\}_{j=1, \ldots, k}$. Thus, $h=\sum_{j=1}^{k} \phi_{j} h$ and $\operatorname{supp}\left(\ell_{j}^{-1} \cdot\left(\phi_{j} h\right)\right) \subset \mathcal{U}_{1}$. By $(5.1)$

$$
\begin{aligned}
\frac{1}{\Gamma_{n}\left(s+\frac{m}{n}\right)} & \left.Z(h, s)\right|_{s=-\frac{m}{n}+q d}=\left.\sum_{j=1}^{k} \frac{1}{\Gamma_{n}\left(s+\frac{m}{n}\right)} Z\left(\phi_{j} h, s\right)\right|_{s=-\frac{m}{n}+q d} \\
& =\left.\sum_{j=1}^{k} \frac{\chi\left(\ell_{j}\right)^{q}}{\Gamma_{n}\left(s+\frac{m}{n}\right)} Z\left(\ell_{j}^{-1} \cdot \phi_{j} h, s\right)\right|_{s=-\frac{m}{n}+q d} \\
& =\sum_{j=1}^{k} \chi\left(\ell_{j}\right)^{q} \int_{\mathcal{O}_{q}}\left(\ell_{j}^{-1} \cdot \phi_{j} h\right)(X) d \nu_{q}(X), \text { by }(5.11) \\
& =\sum_{j=1}^{k} \int_{\mathcal{O}_{q}}\left(\phi_{j} h\right)(X) d \nu_{q}(X) \\
& =\int_{\mathcal{O}_{q}} h(X) d \nu_{q}(X)
\end{aligned}
$$

It now follows that

$$
\left.\frac{1}{\Gamma_{n}\left(s+\frac{m}{n}\right)} Z(h, s)\right|_{s=-\frac{m}{n}+q d}-\nu_{q}
$$

is a distribution supported at $\{0\}$. Lemma 5.2 shows that no (nonzero) distribution supported at $\{0\}$ satisfies the necessary homogeneity property. This concludes the proof.

The main theorem of this section is the following.

Theorem 5.12. For each $h \in \mathcal{S}(\mathfrak{n})$

$$
\frac{1}{\Gamma_{n}\left(s+\frac{m}{n}\right)} Z(h, s)
$$

is an entire function of $s$ and defines a family of tempered distributions. For $q=0,1,2, \ldots, n-1$

$$
\left.\frac{1}{\Gamma_{n}\left(s+\frac{m}{n}\right)} Z(h, s)\right|_{s=q d-\frac{m}{n}}=\nu_{q} .
$$

Proof. In place of (5.13) we consider

$$
\frac{1}{\Gamma_{n}(s)} Z\left(h, s-\frac{m}{n}\right) .
$$

By the general discussion in Appendix B this is an analytic family of tempered distributions for $s \notin S \equiv$ $\{j d-2 l\} \cup\{j d+(e+1)-2 l\}$ with $j=0,1, \ldots, n-1$ and $l \in \mathbf{Z}_{+}$. Furthermore, for $s$ outside the interval $[e+1,(n-1) d](5.14)$ is analytic by Cor. 4.26. By

$$
\frac{Z\left(\nabla^{2 k} h, s-\frac{m}{n}\right)}{\Gamma_{n}(s)}=\left(\prod_{j=0}^{n-1} \prod_{l=0}^{k-1} \frac{s-j d+2 l}{2}\right) \cdot \frac{Z\left(h, s+2 k-\frac{m}{n}\right)}{\Gamma_{n}(s+2 k)}
$$

we have

$$
\frac{Z\left(h, s-\frac{m}{n}\right)}{\Gamma_{n}(s)}=\frac{1}{\left(\prod_{j=0}^{n-1} \prod_{l=0}^{k-1} \frac{s-j d+2 l}{2}\right)} \frac{Z\left(\nabla^{2 k} h, s-2 k-\frac{m}{n}\right)}{\Gamma_{n}(s-2 k)} .
$$

Choosing $k$ big enough so that $s-2 k<e+1$ we see that (5.14) is analytic away from $S^{\prime}=\{j d-2 l\}$. Therefore we need only check analyticity at points $s$ in $S^{\prime} \cap[e+1,(n-1) d]$.

By Cor. B.7 all we need to show is that

$$
\left.\frac{1}{\Gamma_{n}(s)} Z\left(h, s-\frac{m}{n}\right)\right|_{s=s_{0}}<\infty
$$


for $s_{0} \in S \cap[e+1,(n-1) d]$ for $h \in C_{0}^{\infty}(\mathfrak{n})$. By Prop. 5.5 this is the case when $s_{0}=j d, j=0,1, \ldots, n-1$ i.e., when

$$
\left.\frac{1}{\Gamma_{n}(s)} Z\left(h, s-\frac{m}{n}\right)\right|_{s=j d}=\nu_{j}
$$

For $d=1$ or $2, S \cap[e+1,(n-1) d]=\{j d: j=0,1, \ldots, n-1\} \cap[e+1,(n-1) d]$ and we are finished.

For $d>2$ note that

$$
\prod_{j=0}^{n-1} \prod_{l=0}^{k-1} \frac{s-j d+2 l}{2}
$$

has no multiple roots for $k=1,2, \ldots, \frac{d}{2}$ (for $d$ even) and $k=1,2, \ldots, d$ (for $d$ odd). Furthermore, $S^{\prime} \cap[e+$ $1,(n-1) d]=\left\{j d+2 k: j=0,1, \ldots, n-1\right.$ and $k=1, \ldots, \frac{d}{2}($ or $\left.d)\right\}$.

Case 1. Suppose $d$ is even and greater than 2 . Let $k=0,1, \ldots, \frac{d}{2}$. Now consider

$$
\frac{Z\left(\nabla^{2 k} h, s-\frac{m}{n}\right)}{\Gamma_{n}(s)}=\left(\prod_{j=0}^{n-1} \prod_{l=0}^{k-1} \frac{s-j d+2 l}{2}\right) \cdot \frac{Z\left(h, s+2 k-\frac{m}{n}\right)}{\Gamma_{n}(s+2 k)}
$$

evaluated at $s=j d$. The left hand side is zero as $\nabla^{2 k} h=0$ on $\mathcal{O}_{j}$. Since the multiplicities of the zeroes of the polynomial on the right hand side are one has

$$
\left.\frac{Z\left(h, s+2 k-\frac{m}{n}\right)}{\Gamma_{n}(s+2 k)}\right|_{s=j d}<\infty .
$$

Therefore (5.14) is finite for $s \in S \cap[e+1,(n-1) d]$.

Case 2. When $d$ is odd and greater than 2 the proof is exactly as above except $k$ ranges from 0 to $d$.

\section{Positivity}

Define a family of distributions on $\mathfrak{n}$ by

$$
R_{s}(h)=\frac{\pi^{\frac{n}{2}\left(-s+\frac{m}{n}\right)}}{\Gamma_{n}\left(-s+\frac{m}{n}\right)} Z(h,-s), \quad h \in \mathcal{S}(\mathfrak{n}) .
$$

The following theorem is mostly a restatement of what has been proved earlier.

Theorem 6.2. For $R_{s}$ as defined above, the following hold.

(1) $R_{s}$ is defined by the convergent integral

$$
R_{s}(h)=\frac{\pi^{\frac{n}{2}\left(-s+\frac{m}{n}\right)}}{\Gamma_{n}\left(-s+\frac{m}{n}\right)} \int_{\mathfrak{n}} h(X) \nabla(X)^{-s} d X
$$

for $\operatorname{Re}(s)<e+1$.

(2) $R_{s}$ is a holomorphic function of $s$ on all of $\mathbf{C}$.

(3) $R_{\frac{m}{n}}=\frac{\pi^{\frac{m}{2}}}{\Gamma_{n}\left(\frac{m}{n}\right)} \delta_{0}$

(4) For $k=0,1,2, \ldots$,

$$
P\left(\partial_{X}\right)^{k} \delta_{0}=\frac{2^{2 n k}}{\pi^{\left(\frac{m}{n}-2 k\right) \frac{n}{2}}} \Gamma_{n}\left(\frac{m}{n}+2 k\right) R_{\frac{m}{n}+2 k} .
$$

(5) For $q=0,1,2, \ldots, n-1, R_{\frac{m}{n}-q d}=\nu_{q}$, a $\chi_{q}$-quasi-invariant measure on $\mathcal{O}_{q}$.

(6) For $h \in C_{c}^{\infty}(\mathfrak{n})$ with $\operatorname{supp}(h) \subset \mathcal{U}_{q}$

$$
R_{s}(h)=R_{s+(n-q) d}^{q}\left(\nabla^{(n-q) d} R_{s}^{n-q}\left(T_{h}\right)\right) .
$$


Our goal is to see precisely when $R_{s}$ is a positive distribution in the sense that $f \geq 0$ implies $R_{s}(f) \geq 0$.

For a given $\mathfrak{n}$ we set

$$
\Xi_{n}=(-\infty, e+1) \cup\left\{\frac{m}{n}-q d: q=0,1,2, \ldots, n-1\right\} .
$$

By the theorem the distributions $R_{s}$ are positive if $s \in \Xi_{n}$. The following includes a converse.

Theorem 6.4. If $R_{s}$ is a positive distribution then $s \in \Xi_{n}$. The distribution $-R_{s}$ is never positive.

Proof. By Proposition C. 4 the theorem holds for the $n=1$ cases. Now assume $n>1$ and the statement holds for $n-1$ in place of $n$.

Case 1. $s=\frac{m}{n}+2 k, k=0,1,2, \ldots$ By Thm. 6.2, part (4)

$$
R_{\frac{m}{n}+2 k}=C_{k} P\left(\partial_{X}\right) \delta_{0}, \quad \text { where } C_{k} \text { is a positive constant. }
$$

This is positive if and only if $k=0$, i.e., $s=\frac{m}{n} \in \Xi_{n}$.

Case 2. $s \in \mathbf{R} \backslash\left\{\frac{m}{n}+2 k: k=0,1,2, \ldots\right\}$. Recall that Lemma 3.14 provides coordinates $\phi: \mathcal{O}_{q}(q) \times \overline{\mathfrak{n}}_{q}^{L} \times$ $\mathfrak{n}_{n-q}^{\prime} \rightarrow \mathcal{U}_{q}$ and take $q=1$. Define a smooth function $h$ as follows. Choose nonnegative (and not identically zero) functions

$$
\begin{aligned}
& \varphi \in C_{c}^{\infty}\left(\mathfrak{n}_{1}\right) \text { with } \operatorname{supp}(\varphi) \subset \mathcal{U}_{1} \\
& \psi \in C_{c}^{\infty}\left(\overline{\mathfrak{n}}_{1}^{L}\right) \\
& \varphi^{\prime} \in C_{c}^{\infty}\left(\mathfrak{n}_{1}^{\prime}\right) .
\end{aligned}
$$

Then

$$
h(x)= \begin{cases}\varphi(X) \psi(u) \varphi^{\prime}\left(X^{\prime}\right), & \text { if } x=\phi\left(X, u, X^{\prime}\right) \\ 0, & \text { if } x \notin \operatorname{image}(\phi)\end{cases}
$$

defines a nonnegative smooth function on $\mathfrak{n}$ of compact support with $\operatorname{supp}(h) \subset \mathcal{U}_{1}$. Furthermore,

$$
T_{h}\left(X, X^{\prime}\right)=c \varphi(X) \varphi^{\prime}\left(X^{\prime}\right), \quad c_{\psi}=\int \psi(u) d u>0 .
$$

It follows from Thm. 6.2, part (6) that

$$
R_{s}(h)=c_{\psi} R_{s-(n-1) d}^{1}\left(\varphi \cdot \nabla_{1}^{d(n-1)}\right) R_{s}^{n-1}\left(\varphi^{\prime}\right) .
$$

Since $\operatorname{supp}(\varphi)$ is compact and away from $\{0\}\left(=\left\{X \in \mathfrak{n}_{1}: \nabla_{1}(X)=0\right\}\right)$,

$$
R_{s-(n-1) d}^{1}\left(\varphi \nabla_{1}^{(n-1) d}\right)=\frac{\pi^{\left(-s+\frac{m}{n}\right) \frac{n}{2}}}{\Gamma_{1}\left(-s+\frac{m}{n}\right)} \int_{\mathfrak{n}_{1}} \varphi(X) \nabla_{1}(X)^{-s+2 d(n-1)} d X
$$

for all $s$. In particular this is nonzero and has the same sign as $\Gamma_{1}\left(-s+\frac{m}{n}\right)$. Therefore, there are positive constants $C_{+}(s)$ so that

$$
R_{s}^{n-1}\left(\varphi^{\prime}\right)=C_{+}(s) \Gamma_{1}\left(-s+\frac{m}{n}\right) R_{s}(h) .
$$

Now suppose that $R_{s}$ is a positive distribution. If $\Gamma_{1}\left(-s+\frac{m}{n}\right)>0$ then $R_{s}^{n-1}\left(\varphi^{\prime}\right)>0$ for all $\varphi^{\prime} \in$ $C_{c}^{\infty}\left(\mathfrak{n}_{n-1}^{\prime}\right)$. Therefore, $R_{s}^{n-1}$ is a positive distribution, so $s \in \Xi_{n-1}$. Therefore, $s \in \Xi_{n}$. However, if $\Gamma_{1}\left(-s+\frac{m}{n}\right)<0$ then $-R_{s}^{n-1}$ is positive which contradicts the inductive hypothesis.

Now suppose $-R_{s}$ is a positive distribution. When $\Gamma_{1}\left(-s+\frac{m}{n}\right)>0,-R_{s}^{n-1}$ will be a positive distribution, a contradiction to the inductive hypothesis. When $\Gamma_{1}\left(-s+\frac{m}{n}\right)<0, R_{s}^{n-1}$ is a positive distribution, so $s \in \Xi_{n-1}$. However, $\Gamma_{1}\left(-s+\frac{m}{n}\right)>0$ when $s \in \Xi_{n-1}\left(\right.$ since $-s+\frac{m}{n}>0$ for $\left.s \in \Xi_{n-1}\right)$. 
Case 3. $s \in \mathbf{C} \backslash \mathbf{R}$. We choose nonnegative functions in a similar manner as above, however the roles of $\mathfrak{n}_{1}$ and $\mathfrak{n}_{n-1}$ are switched (that is, we take $q=n-1$ ). Let

$$
\begin{aligned}
& \varphi \in C_{c}^{\infty}\left(\mathfrak{n}_{n-1}\right) \text { with } \operatorname{supp}(\varphi) \subset \mathcal{O}_{n-1}(n-1), \\
& \psi \in C_{c}^{\infty}\left(\overline{\mathfrak{n}}_{q}^{L}\right) \text { with } c_{\psi}=\int \psi(u) d u>0, \\
& \varphi_{1}, \varphi_{2} \in C_{c}^{\infty}\left(\mathfrak{n}_{1}\right) \text { such that } \frac{R_{s}^{1}\left(\varphi_{1}\right)}{R_{s}^{1}\left(\varphi_{2}\right)}=\alpha_{s} \in \mathbf{C} \backslash \mathbf{R} .
\end{aligned}
$$

Note that since $\varphi$ has compact support in $\mathcal{O}_{n-1}(n-1)$

$$
R_{s-d}^{n-1}\left(\nabla_{n-1} \varphi\right)=\frac{\pi^{\frac{n}{2}\left(-s+\frac{m}{n}\right)}}{\Gamma_{n-1}\left(-s+\frac{m}{n}\right)} \int_{\mathfrak{n}_{n-1}} \varphi(X) \nabla_{n-1}(X)^{-s+d} d X
$$

for all $s \in \mathbf{C}$. Since we are in the case where $s \in \mathbf{C} \backslash \mathbf{R}, \Gamma_{n-1}\left(-s+\frac{m}{n}\right)$ has no poles. Therefore, $c_{\varphi} \equiv$ $R_{s-d}^{n-1}\left(\nabla_{n-1}^{d} \cdot \varphi\right) \neq 0$.

To see that functions $\varphi_{1}$ and $\varphi_{2}$ exist note that the functions $\tilde{\varphi}_{1}\left(X^{\prime}\right)=\left\|X^{\prime}\right\|^{2} \mathrm{e}^{-\left\|X^{\prime}\right\|^{2}}$ and $\tilde{\varphi}_{2}\left(X^{\prime}\right)=$ $\mathrm{e}^{-\left\|X^{\prime}\right\|^{2}}, X^{\prime} \in \mathfrak{n}_{1}$ are Schwartz functions so that

$$
\frac{R_{s}^{1}\left(\tilde{\varphi}_{1}\right)}{R_{s}^{1}\left(\tilde{\varphi}_{2}\right)}=\frac{1}{2}(-s+e+1)
$$

by (C.5). We may take $\varphi_{1}$ and $\varphi_{2}$ to be compactly supported functions approximating $\tilde{\varphi}_{1}$ and $\tilde{\varphi}_{2}$ sufficiently closely.

Again using the coordinates $\phi:\left(X, u, X^{\prime}\right) \rightarrow \exp (u)\left(X+X^{\prime}\right)$ we define smooth compactly supported functions by

$$
h_{i}(x)= \begin{cases}\varphi(X) \psi(u) \varphi_{i}\left(X^{\prime}\right), & \text { if } x=\phi\left(X, u, X^{\prime}\right) \\ 0, & \text { if } x \notin \operatorname{image}(\phi)\end{cases}
$$

for $i=1,2$. By Thm. 6.2, part (6) we have

$$
R_{s}\left(h_{i}\right)=c_{\psi} c_{\varphi} R_{s}^{1}\left(\varphi_{i}\right)
$$

Now $R_{s}\left(h_{i}\right)$ cannot be positive (or negative) for both $i=1$ and $i=2$ since

$$
\frac{R_{s}\left(h_{1}\right)}{R_{s}\left(h_{2}\right)}=\frac{R_{s}^{1}\left(\varphi_{1}\right)}{R_{s}^{1}\left(\varphi_{2}\right)}=\alpha_{s} \in \mathbf{C} \backslash \mathbf{R}
$$

Therefore, $\pm R_{s}$ is not a positive distribution for $s \in \mathbf{C} \backslash \mathbf{R}$.

\section{Generalized PRINCIPAL SERIES REPRESENTATIONS}

For each $s \in \mathbf{C}$ there is a normalized principal series representation

$$
\operatorname{Ind}_{P}^{G}(s)=\left\{\varphi: G \rightarrow \mathbf{C} \mid \varphi \text { is smooth and } \varphi(\text { gman })=\mathrm{e}^{-\left(s+\frac{m}{n}\right) \Lambda_{0}(\log (a))} \varphi(g), \text { man } \in P=M A N\right\} .
$$

The group $G$ acts by left translation:

$$
(g \cdot \varphi)\left(g_{1}\right)=\varphi\left(g^{-1} g_{1}\right) .
$$

This principal series representation may be realized as smooth functions on $\overline{\mathfrak{n}}$ as follows. Write $\bar{n}_{Y} \equiv \exp (Y)$ for $Y \in \overline{\mathfrak{n}}$. For $\varphi \in \operatorname{Ind}_{P}^{G}(s)$ set

$$
f(Y)=\varphi\left(\bar{n}_{Y}\right), Y \in \overline{\mathfrak{n}} .
$$

Then $\operatorname{Ind}_{P}^{G}(s)$ may be identified with

$$
I(s)=\left\{f \in C^{\infty}(\overline{\mathfrak{n}}): f(Y)=\varphi\left(\bar{n}_{Y}\right), \text { for some } \varphi \in \operatorname{Ind}_{P}^{G}(s)\right\} .
$$


Since $\bar{N} P$ is dense in $G$ and any $g \in \bar{N} P$ has a unique decomposition as

$$
g=\bar{n}(g) m(g) a(g) n(g) \in \bar{N} M A N,
$$

the action is given by

$$
(g \cdot f)(Y)=\mathrm{e}^{-\left(s+\frac{m}{n}\right) \Lambda_{0}}\left(a\left(g^{-1} \bar{n}_{Y}\right)\right) f\left(\log \left(\bar{n}\left(g^{-1} \bar{n}_{Y}\right)\right)\right) .
$$

In particular

$$
\begin{aligned}
& (\ell \cdot f)\left(Y_{1}\right)=\mathrm{e}^{-\left(s+\frac{m}{n}\right) \Lambda_{0}}(a(\ell)) f\left(\ell^{-1} \cdot Y_{1}\right) \\
& \left(\bar{n}_{Y} \cdot f\right)\left(Y_{1}\right)=f\left(Y_{1}-Y\right) .
\end{aligned}
$$

For each $s$ with $\operatorname{Re}(s)>d(n-1)$ there is a $G$-intertwining operator

$$
\tilde{A}_{s}: I(s) \rightarrow I(-s)
$$

which is given by the convergent integral

$$
\left(\tilde{A}_{s} f\right)(Y)=\int_{\overline{\mathrm{n}}} \varphi\left(\bar{n}_{Y} w \bar{n}_{Y_{1}}\right) d Y_{1} .
$$

The form we will use is easily derived from this ([10], pages 183 and 200):

$$
\begin{gathered}
\left(\tilde{A}_{s} f\right)(Y)=\int_{\overline{\mathfrak{n}}} \bar{\nabla}\left(Y_{1}\right)^{s-\frac{m}{n}} f\left(Y+Y_{1}\right) d Y_{1} \\
=\int_{\overline{\mathfrak{n}}} \bar{\nabla}\left(Y_{1}-Y\right)^{s-\frac{m}{n}} f\left(Y_{1}\right) d Y_{1} .
\end{gathered}
$$

The integral converges for $\operatorname{Re}(s)>d(n-1)$ (by Lemma 3.16 and by Lemma 3.15).

Note that the Schwartz space $\mathcal{S}(\overline{\mathfrak{n}})$ is contained in $I(s)$ for all $s$. Also, for each $f \in I(s)$ there is a constant $C$ so that

$$
|f(Y)| \leq C \mathrm{e}^{-\left(\operatorname{Re}(s)+\frac{m}{n}\right) \Lambda_{0}\left(H\left(\bar{n}_{Y}\right)\right)},
$$

for all $Y \in \overline{\mathfrak{n}}$. In particular,

$$
I(s) \subset L^{2}\left(\overline{\mathfrak{n}}, \mathrm{e}^{2 \operatorname{Re}(s) \Lambda_{0}\left(H\left(\bar{n}_{Y}\right)\right)} d Y\right) .
$$

The intertwining operators $\tilde{A}_{s}$ are complex analytic in $s$ for $\operatorname{Re}(s)>d(n-1)$ and have meromorphic continuations to all of $\mathbf{C}$. This is a well-known general fact (see, for example, [10] and [26]). However one can see this directly for Schwartz functions as follows.

$$
\tilde{A}_{s}\left(\bar{P}\left(\partial_{Y}\right) f\right)(Y)=\int_{\overline{\mathfrak{n}}} f\left(Y+Y_{1}\right) \bar{P}\left(\partial_{Y}\right) \bar{\nabla}\left(Y_{1}\right)^{s-\frac{m}{n}} d Y_{1}=b\left(s-\frac{m}{n}\right)\left(\tilde{A}_{s-2} f\right)(Y),
$$

so the argument of Section 4 applies. Define

$$
A_{s}(f)=\frac{\pi^{\frac{n s}{2}}}{\Gamma_{n}(s)} \tilde{A}_{s}(f) .
$$

For $s \in \mathbf{R}$ there is a $G$-invariant hermitian form on $I(s)$ defined by

$$
\left\langle f_{1}, f_{2}\right\rangle=\int_{\overline{\mathfrak{n}}} f_{1}(Y) \overline{A_{s} f_{2}(Y)} d Y .
$$

See [10, Prop. 14.23], for example. Also, there is a well-defined invariant hermitian form on the image of $A_{s}$ in $I(-s)$ given by

$$
\left\langle A_{s} f_{1}, A_{s} f_{2}\right\rangle_{s}=\left\langle f_{1}, f_{2}\right\rangle .
$$

Consider Schwartz functions $f, f_{1}$ and $f_{2}$ on $\mathcal{S}(\overline{\mathfrak{n}})$. Let $\tau_{Y} f=f(\cdot+Y)$. Then for $\operatorname{Re}(s) \gg 0$

$$
\left(A_{s} f\right)(Y)=\frac{\pi^{\frac{n s}{2}}}{\Gamma_{n}(s)} \overline{\mathbf{Z}}\left(\tau_{Y} f, s-\frac{m}{n}\right),
$$


by the definitions as integrals. This holds for all $s$ since both sides are meromorphic in $s$. By Theorem 4.4

$$
\begin{aligned}
& \left(A_{s} f\right)(Y)=\frac{\pi^{\frac{n}{2}\left(-s+\frac{m}{n}\right)}}{\Gamma_{n}\left(-s+\frac{m}{n}\right)} \mathbf{Z}\left(\left(\tau_{Y} f\right)^{\wedge},-s\right) \\
& =R_{s}\left(\left(\tau_{Y} f\right)^{\widehat{ }}\right) \text {. }
\end{aligned}
$$

Therefore, for $s \ll 0$

$$
\begin{aligned}
\left\langle A_{s} f_{1}, A_{s} f_{2}\right\rangle_{s} & =\int_{\overline{\mathfrak{n}}} f_{1}(Y) \overline{R_{s}\left(\left(\tau_{Y} f\right)^{\wedge}\right)} d Y \\
& =\frac{\pi^{\frac{n}{2}\left(-s+\frac{m}{n}\right)}}{\Gamma_{n}\left(-s+\frac{m}{n}\right)} \int_{\overline{\mathfrak{n}}} \int_{\mathfrak{n}} f_{1}(Y) \nabla(X)^{-s} \mathrm{e}^{2 \pi i\langle X, Y\rangle} \overline{\widehat{f}_{2}}(X) d X d Y \\
& =\frac{\pi^{\frac{n}{2}\left(-s+\frac{m}{n}\right)}}{\Gamma_{n}\left(-s+\frac{m}{n}\right)} \int_{\mathfrak{n}} \nabla(X)^{-s} \widehat{f_{1}}(X) \overline{\widehat{f}_{2}}(X) d X \\
& =R_{s}\left(\left(f_{1} * \overline{f_{2}}\right)^{-}\right) .
\end{aligned}
$$

Since both sides are real analytic in $s$ we have proved the following proposition.

Proposition 7.5. For $s \in \mathbf{R}$ and $f_{1}, f_{2} \in \mathcal{S}(\overline{\mathfrak{n}})$

$$
\left\langle A_{s} f_{1}, A_{s} f_{2}\right\rangle_{s}=R_{s}\left(\left(f_{1} * \overline{f_{2}}\right) \widehat{)}\right)=R_{s}\left(\widehat{f_{1}} \overline{\hat{f}_{2}}\right) .
$$

Proposition 7.7. When $s \notin \Xi_{n}$ the hermitian form $\langle,\rangle_{s}$ on $\operatorname{Im}\left(A_{s}\right)$ is not positive definite (so the ( $\left.\mathfrak{g}, K\right)$ module of $\operatorname{Im}\left(A_{s}\right)$ is not unitarizable).

Proof. The distribution $R_{s}$ is positive if and only if the right hand side of (7.6) is positive when $f_{1}=f_{2}$.

Proposition 7.8. ([20, Theorems $4 \mathrm{~A}$ and $4 \mathrm{~B}])$ For $G \neq S O(p, q)$ (case 4 on tables 1 and 2) the principal series representation $I(s)$ satisfies

(1) $I(s)$ is irreducible and unitarizable for $|s|<e+1$, and

(2) $I(s)$ contains a unitarizable quotient for $s=\frac{m}{n}-d q$ for $q=0,1, \ldots, n-1$.

Remark 7.9. Considering $s \geq 0$, the points of possible unitarizability of $\operatorname{Im}\left(A_{s}\right)$ are

$$
\Xi_{n} \cap[0, \infty)=[0, e+1) \cup\left\{\frac{m}{n}-q d: q=0,1, \ldots, n-1\right\}
$$

pictured as the following set:

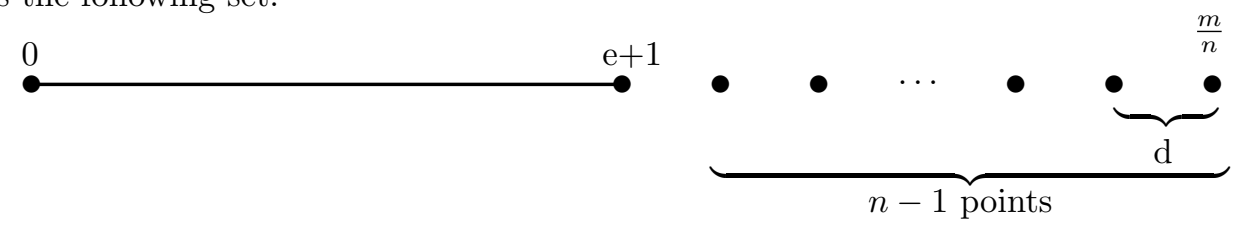

\section{UNITARY REALIZATION}

For this section we assume the $G \neq S O(p, q)$ and we consider only $s \geq 0$. Consider the image of $A_{s}$ in $I(-s)$. $\operatorname{Im}\left(A_{s}\right)$ is $G$-invariant and (7.3) defines a $G$-invariant hermitian form on $\operatorname{Im}\left(A_{s}\right)$. By Prop. 7.8, this form is positive definite (on the $K$-finite vectors) for

$$
s \in \Xi_{n} .
$$

For $s$ satisfying (8.1) define

$$
\mathcal{H}_{s}^{0}=\operatorname{Im}\left(A_{s}\right),
$$

a pre-hilbert space with inner product $\langle,\rangle_{s}$. Let $\mathcal{H}_{s}$ be the completion. Then $\mathcal{H}_{s}$ is a unitary representation of $G$. 
Lemma 8.2. $A_{s}(\mathcal{S}(\overline{\mathfrak{n}})) \subset \mathcal{H}_{s}$ is dense.

Proof. We check that $A_{s}(\mathcal{S}(\overline{\mathfrak{n}}))^{\perp}=0$. Suppose that $f_{2} \in I(s)$ and $A_{s} f_{2} \perp A_{s}(\mathcal{S}(\overline{\mathfrak{n}}))$ i.e., for all $f_{1} \in \mathcal{S}(\overline{\mathfrak{n}})$

$$
0=\left\langle A_{s} f_{1}, A_{s} f_{2}\right\rangle_{s}=\int_{\overline{\mathrm{n}}} f_{1}(Y) \overline{A_{s} f_{2}(Y)} d Y .
$$

By $(7.1)$

$$
f_{1} \in L^{2}\left(\overline{\mathfrak{n}}, \mathrm{e}^{2 \operatorname{Re}(s) \Lambda_{0}\left(H\left(\bar{n}_{Y}\right)\right)} d Y\right) \text { and } A_{s} f_{2} \in L^{2}\left(\overline{\mathfrak{n}}, \mathrm{e}^{-2 \operatorname{Re}(s) \Lambda_{0}\left(H\left(\bar{n}_{Y}\right)\right)} d Y\right) .
$$

Therefore,

$$
0=\int_{\overline{\mathfrak{n}}}\left(f_{1}(Y) \mathrm{e}^{-s \Lambda_{0}\left(H\left(\bar{n}_{Y}\right)\right)}\right)\left(A_{s} f_{2}(Y) \mathrm{e}^{s \Lambda_{0}\left(H\left(\bar{n}_{Y}\right)\right)}\right) d Y,
$$

where the integrand is now written as a product of two functions in $L^{2}(\overline{\mathfrak{n}}, d Y)$. Since $\mathrm{e}^{-s \Lambda_{0}\left(H\left(\bar{n}_{Y}\right)\right)}$ is a power of a nonzero polynomial $\left\{f_{1}(Y) \mathrm{e}^{-s \Lambda_{0}\left(H\left(\bar{n}_{Y}\right)\right)}: f_{1} \in \mathcal{S}(\overline{\mathfrak{n}})\right\}$ is dense in $L^{2}(\overline{\mathfrak{n}}, d Y)$. We now conclude that $A_{s} f_{2}=0$. The lemma follows from the fact that $\mathcal{H}_{s}^{0}$ is dense in $\mathcal{H}_{s}$.

For the unitary realizations of the $I_{s}$ we treat the cases of $s \in[0, e+1)$ and $s=\frac{m}{n}-q d, d=0,1, \ldots, n-1$ separately because the distributions $R_{s}$ have very different forms in these two cases.

Case 1: $s \in[0, e+1)$. For $s \in[0, e+1), R_{\frac{m}{n}-s}$ is given by a convergent integral. Therefore by (7.4) and Prop. 7.5

$$
\begin{aligned}
A_{s} f(Y) & =\frac{\pi^{\frac{n}{2}\left(-s+\frac{m}{n}\right)}}{\Gamma_{n}\left(-s+\frac{m}{n}\right)} \int_{\mathfrak{n}} \mathrm{e}^{-s \pi i\langle X, Y\rangle} \widehat{f}(X) \nabla(X)^{-s} d X \\
& =\frac{\pi^{\frac{n}{2}\left(-s+\frac{m}{n}\right)}}{\Gamma_{n}\left(-s+\frac{m}{n}\right)}\left(\widehat{f} \nabla(\cdot)^{-s}\right)^{\wedge}(Y)
\end{aligned}
$$

and

$$
\left\langle A_{s} f_{1}, A_{s} f_{2}\right\rangle_{s}=\frac{\pi^{\frac{n}{2}\left(-s+\frac{m}{n}\right)}}{\Gamma_{n}\left(-s+\frac{m}{n}\right)} \int_{\mathfrak{n}} \widehat{f}_{1}(X) \overline{\widehat{f}_{2}(X)} \nabla(X)^{-s} d X
$$

for all Schwartz functions $f, f_{1}$ and $f_{2}$ on $\overline{\mathfrak{n}}$.

The Schwartz space $\mathcal{S}(\mathfrak{n})$ has a natural action by $\bar{P}=L \bar{N}$ via the Fourier transform as follows. Let $\vee$ denote the inverse Fourier transform. For $h \in \mathcal{S}(\mathfrak{n})$ define

$$
(\bar{p} \cdot h)(X)=\left(\bar{p} \cdot h^{\vee}\right)^{\wedge}(X) .
$$

Then

$$
\begin{aligned}
& (\ell \cdot h)(X)=\mathrm{e}^{\left(s-\frac{m}{n}\right) \Lambda_{0}}(a(\ell)) h(\ell \cdot X) \\
& \left(\bar{n}_{Y} \cdot h\right)(X)=\mathrm{e}^{-2 \pi i\langle X, Y\rangle} h(X) .
\end{aligned}
$$

Theorem 8.6. For $s \in[0, e+1)$, let $d_{s} X=\frac{\pi^{\frac{n}{2}\left(-s+\frac{m}{n}\right)}}{\Gamma_{n}\left(-s+\frac{m}{n}\right)} d X$. Then $\mathcal{H}_{s}$ is unitarily equivalent to $L^{2}\left(\mathfrak{n}, \nabla(X)^{-s} d_{s} X\right)$ as $\bar{P}$-representations. This representation is irreducible.

Proof. Temporarily set $V_{s}=A_{s}(\mathcal{S}(\overline{\mathfrak{n}}))$. Then

$$
V_{s}=\left\{\left(h \nabla(X)^{-s} d X\right)^{\wedge}: h \in \mathcal{S}(\mathfrak{n})\right\}
$$

by (7.4). We have $\mathcal{S}(\mathfrak{n}) \subset L^{2}\left(\mathfrak{n}, \nabla(X)^{-s} d_{s} X\right)$ and $V_{s} \subset \mathcal{H}_{s}$ as dense subspaces (by Lemma 8.2). Consider

$$
T: \mathcal{S}(\mathfrak{n}) \rightarrow V_{s}
$$


defined by

$$
T(h)=\left(h \cdot \nabla(\cdot)^{-s}\right)^{\wedge} .
$$

We will check that $T$ is an isometry onto a dense subset of $\mathcal{H}_{s}$ and is $\bar{P}$-equivariant, then $T$ will extend to a unitary equivalence of unitary $\bar{P}$-representations.

Let $h \in \mathcal{S}(\mathfrak{n})$.

$$
\begin{aligned}
\langle T(h), T(h)\rangle_{s} & =\left\langle A_{s}\left(h^{\vee}\right), A_{s}\left(h^{\vee}\right)\right\rangle_{s}, \text { by }(8.3) \\
& =\int_{\mathfrak{n}}|h(X)|^{2} \nabla(X)^{-s} d_{s} X \text {, by }(8.4) .
\end{aligned}
$$

(Note that $R_{s}$ is given by a convergent integral for $s \in[0, e+1)$.) The equivariance follows easily:

$$
T(\bar{p} \cdot h)=T\left(\left(\bar{p} \cdot h^{\vee}\right)^{\wedge}\right)=A_{s}\left(\bar{p} \cdot h^{\vee}\right)=\bar{p} \cdot A_{s}\left(h^{\vee}\right)=\bar{p} \cdot T(h) .
$$

The irreducibility is a standard application of Schur's Lemma as follows. Any $\bar{N}$-intertwining operator of $L^{2}\left(\mathfrak{n}, \nabla(X)^{-s} d_{s} X\right)$ must be multiplication by a bounded function. Transitivity (up to measure zero) of $L$ on $\mathfrak{n}$ shows that if the operator is also $L$-intertwining then the bounded function must be a constant (a.e.).

Corollary 8.7. The $\bar{P}$-representation $L^{2}\left(\mathfrak{n}, \nabla(X)^{-s} d_{s} X\right)$ extends to an irreducible unitary representation of $G$.

Case 2: $s=\frac{m}{n}-q d, q=0,1, \ldots, n-1$. Define

$$
\mathcal{F}_{R}(f)=\left.\widehat{f}\right|_{\mathcal{O}_{q}}, \text { for } f \in \mathcal{S}(\overline{\mathfrak{n}})
$$

and

$$
\mathcal{F}_{E}(h)=\left(h d \nu_{q}\right)^{\wedge}, \text { for } h \in \mathcal{F}_{R} \mathcal{S}(\mathfrak{n}) .
$$

Then by (7.4) and (7.6)

$$
A_{s}(f)=\mathcal{F}_{E} \mathcal{F}_{R}(f), f \in \mathcal{S}(\overline{\mathfrak{n}}),
$$

and

$$
\left\langle A_{s} f_{1}, A_{s} f_{2}\right\rangle_{s}=\int_{\mathcal{O}_{q}} \widehat{f_{1}} \overline{\widehat{f}_{2}} d \nu_{q} .
$$

As in Case 1, we may define a $\bar{P}$-action on $L^{2}\left(\mathcal{O}_{q}, d \nu_{q}\right)$ via the Fourier transform. Letting $h \in L^{2}\left(\mathcal{O}_{q}, d \nu_{q}\right)$ and $s=\frac{m}{n}-d q$ we define

$$
\begin{aligned}
& (\ell \cdot h)(X)=e^{-d q \Lambda_{0}}(a(\ell)) h\left(\ell^{-1} \cdot X\right)=\chi_{d}(\ell)^{-\frac{1}{2}} h\left(\ell^{-1} \cdot X\right) \\
& \left(\bar{n}_{Y} \cdot h\right)(X)=\mathrm{e}^{-2 \pi i\langle X, Y\rangle} h(X) .
\end{aligned}
$$

Lemma 8.10. Let $q=0,1, \ldots, n-1$.

(1) $L^{2}\left(\mathcal{O}_{q}, d \nu_{q}\right)$ is an irreducible unitary representation of $\bar{P}$.

(2) $\mathcal{F}_{R}: \mathcal{S}(\overline{\mathfrak{n}}) \rightarrow L^{2}\left(\mathcal{O}_{q}, d \nu_{q}\right)$ is $\bar{P}$-equivariant and has dense image. 
Proof. For unitarity note that $\left\|\bar{n}_{Y} \cdot h\right\|_{\mathcal{O}_{q}}^{2}=\|h\|^{2}$ is clear from (8.5) and

$$
\begin{aligned}
\|\ell \cdot h\|_{\mathcal{O}_{q}}^{2} & =\int_{\mathcal{O}_{q}}\|\ell \cdot h\|^{2} d \nu_{q} \\
& =\int_{\mathcal{O}_{q}} \| \chi_{d}(\ell)^{-\frac{1}{2}} h\left(\ell^{-1} \cdot X \|^{2} d \nu_{q}(X)\right. \\
& =\int_{\mathcal{O}_{q}}\|h(X)\|^{2} d \nu_{q}(X), \text { by Cor. } 3.7 .
\end{aligned}
$$

Irreducibility is as in the proof of Theorem 8.6. The $\bar{P}$-equivariance of $\mathcal{F}_{R}$ is also as in the proof of Theorem 8.6. The image of $\mathcal{F}_{R}$ is dense by irreducibility of $L^{2}\left(\mathcal{O}_{q}, d \nu_{q}\right)$.

Theorem 8.11. $\mathcal{F}_{E}$ extends to a $\bar{P}$-equivariant unitary equivalence between $L^{2}\left(\mathcal{O}_{q}, d \nu_{q}\right)$ and $\mathcal{H}_{\frac{m}{n}}-q d, q=$ $0,1, \ldots, n-1$.

Proof. $\mathcal{F}_{E}: \mathcal{F}_{R}(\mathcal{S}(\overline{\mathfrak{n}})) \rightarrow \mathcal{H}_{\frac{m}{n}-q d}^{0}$ is an isometry by (8.8) and (8.9). By part (2) of Lemma 8.10, $\mathcal{F}_{E}$ extends to a unitary equivalence of $L^{2}\left(\mathcal{O}_{q}, d \nu_{q}\right)$ and $\mathcal{H}_{\frac{m}{n}-d q}=\overline{\mathcal{H}_{\frac{m}{n}}^{0}-d q}$. We now check the $\bar{P}$-equivariance of $\mathcal{F}_{E}$ on $\mathcal{F}_{R}(\mathcal{S}(\overline{\mathfrak{n}}))$. Let $h=\mathcal{F}_{R}(f) \in \mathcal{F}_{R}(\mathcal{S}(\mathfrak{n}))$.

$$
\mathcal{F}_{E}(\bar{p} \cdot h)=\mathcal{F}_{E}\left(\bar{p} \cdot \mathcal{F}_{R} f\right)=\mathcal{F}_{E} \mathcal{F}_{R}(\bar{p} \cdot f) A_{s}(\bar{p} \cdot f)=\bar{p} \cdot A_{s} f=\mathcal{F}_{E}\left(\mathcal{F}_{R} f\right)=\mathcal{F}_{E}(h)
$$

Corollary 8.12. The $\bar{P}$-representations $L^{2}\left(\mathcal{O}_{q}, d \nu_{q}\right)$ extend to irreducible unitary representations of $G$.

\section{Appendix A. TABles}

The following two tables give information on the groups under consideration in this paper.

\begin{tabular}{|l|c|c|c|c|c|}
\hline & $G$ & $n=\operatorname{rank}(\mathfrak{n})$ & $m=\operatorname{dim}(\mathfrak{n})$ & $d$ & $e$ \\
\hline 1. & $G L(2 n, \mathbf{R}), n \geq 2$ & $n$ & $n^{2}$ & 1 & 0 \\
2. & $O(2 n, 2 n), n \geq 2$ & $n$ & $n(2 n-1)$ & 2 & 0 \\
3. & $E_{7}(7)$ & 3 & 27 & 4 & 0 \\
4. & $O(p, q), p, q \geq 3$ & 2 & $p+q-2$ & $(p+q-4) / 2$ & 0 \\
\hline 5. & $S p(n, \mathbf{C})$ & $n$ & $n(n+1)$ & 1 & 1 \\
6. & $S L(2 n, \mathbf{C})$ & $n$ & $2 n^{2}$ & 2 & 1 \\
7. & $S O(4 n, \mathbf{C})$ & $n$ & $2 n(2 n-1)$ & 4 & 1 \\
8. & $E_{7, \mathbf{C}}$ & 3 & 54 & 8 & 1 \\
9. & $S O(p, \mathbf{C})$ & 2 & $2(p-2)$ & $p-4$ & 1 \\
\hline 10. & $S p(n, n)$ & $n$ & $n(2 n+1)$ & 2 & 2 \\
11. & $S L(2 n, \mathbf{H})$ & $n$ & $4 n^{2}$ & 4 & 3 \\
12. & $S O(p, 1)$ & 1 & $p$ & 0 & $p-1$ \\
\hline
\end{tabular}

TABle 1 


\begin{tabular}{|c|c|c|c|}
\hline & $V \simeq \mathfrak{n}$ & $L$ & $\nabla$ \\
\hline 1. & $M(n \times n, \mathbf{R})$ & $G L(n, \mathbf{R}) \times G L(n, \mathbf{R})$ & $|\operatorname{det}|$ \\
\hline 2. & $\operatorname{Skew}(2 n: \mathbf{R})$ & $G L(2 n, \mathbf{R})$ & Pfaffian \\
\hline 3. & $\operatorname{Herm}\left(3, \mathbf{O}_{\text {split }}\right)$ & $E_{6}(6) \times \mathbf{R}^{\times}$ & degree 3 real polynomial \\
\hline 4. & $\mathbf{R}^{p-1, q-1}$ & $\mathbf{R}^{\times} S O(p-1, q-1)$ & $(X, X)$ \\
\hline 5. & $\operatorname{Sym}(n, \mathbf{C})$ & $G L(n: \mathbf{C})$ & $|\operatorname{det}|$ \\
\hline 6. & $M(n \times n, \mathbf{C})$ & $S(G L(n, \mathbf{C}) \times G L(n, \mathbf{C}))$ & det $\mid$ \\
\hline 7 . & $\operatorname{Skew}(2 n, \mathbf{C})$ & $G L(2 n, \mathbf{C})$ & |Pfaffian $\mid$ \\
\hline 8. & $\operatorname{Herm}(3, \mathbf{O})_{\mathbf{C}}$ & $E_{6, \mathbf{C}} \mathbf{C}^{\times}$ & |degree 3 complex poly| \\
\hline 9. & $\mathbf{C}^{p-1}$ & $O(p-2: \mathbf{C}) \times \mathbf{C}^{\times}$ & $|(Z, Z)|$ \\
\hline 10. & $\operatorname{Sym}(2 n, \mathbf{C}) \cap M(n \times n, \mathbf{H})$ & $G L(n, \mathbf{H})$ & $\left|\operatorname{det}_{\mathbf{C}}(Z)\right|^{\frac{1}{2}}$ \\
\hline 11. & $M(n \times n, \mathbf{H})$ & $G L(n, \mathbf{H}) \times G L(n, \mathbf{H})$ & $\left|\operatorname{det}_{\mathbf{C}}(Z)\right|^{\frac{1}{2}}$ \\
\hline 12. & $\mathbf{R}^{p, 1}$ & $O(p-1) \times \mathbf{R}^{\times}$ & $\|\cdot\|$ \\
\hline
\end{tabular}

TABLE 2. Jordan algebras for the groups in Table 1.

Remark A.1. For Cases 10 and 11 we view the quaternionic matrices as complex matrices of the form $Z=\left(\begin{array}{cc}A & B \\ -\bar{B} & \bar{A}\end{array}\right)$. Then $\operatorname{det}_{\mathbf{C}}$ refers to the determinant of the complex matrix.

\section{Appendix B. Meromorphic families of Distributions}

We give a few (well-known) general facts about meromorphic families of distributions. Suppose $m(x)$ is a positive polynomial of degree $m$ on $\mathbf{R}^{n}$ with Bernstein polynomial $b(s)$ defined by $m\left(\partial_{x}\right) m(x)^{\frac{s}{2}}=$ $b(s) m(x)^{\frac{s}{2}-1}$. Let $\left\{\alpha_{j}\right\}$ be the roots of $b(s)$ and set $S=\left\{\alpha_{j}-2 l: l \in \mathbf{Z}_{+}\right\}$. Define, for $h \in \mathcal{S}\left(\mathbf{R}^{n}\right)$,

$$
T_{s}(h)=\int_{\mathbf{R}^{n}} h(x) m(x)^{\frac{s}{2}} d x,
$$

a convergent integral for $\operatorname{Re}(s)>0$. For $\operatorname{Re}(s)>0, T_{s}(h)$ is a complex analytic function of $s$. From the formula

$$
T_{s}(h)=\frac{1}{b(s+2) b(s+4) \cdots b(s+2 k)} T_{s+k}\left(m\left(\partial_{x}\right)^{k} h(x)\right)
$$

it follows that $T_{s}(h)$ has a meromorphic continuation to all of $\mathbf{C}$ with possible poles in $S$.

Lemma B.2. Consider the Laurent expansion of $T_{s}(h)$ for an arbitrary $s_{0} \in \mathbf{C}$ :

$$
T_{s}(h)=\sum_{m \geq-d}^{\infty} T^{m}(h)\left(s-s_{0}\right)^{m}
$$

for some $d=0,1,2, \ldots$ Then each $T^{m}$ is a tempered distribution.

Proof. Let $N \in \mathbf{Z}_{+}$and let $\|\cdot\|_{N}$ be the Schwartz norm

$$
\|h\|_{N}=\sup \left\{(1+|x|)^{N}|h(x)|\right\} .
$$

In particular, $|h(x)| \leq\|h\|_{N}(1+|x|)^{-N}$ for all $x \in \mathbf{R}^{n}$. Choose a constant $C^{\prime}$ so that

$$
|m(x)| \leq C^{\prime}(1+|x|)^{M}, \quad x \in \mathbf{R}^{N} .
$$

Therefore,

$$
\left|h(x) m(x)^{\frac{s}{2}}\right| \leq C^{\prime}|| h \|_{N}(1+|x|)^{-\left(N-\operatorname{Re}\left(\frac{s}{2}\right) M\right)} \text { for } \operatorname{Re} s>0 .
$$


For any $s_{1}$ with $\operatorname{Re}\left(s_{1}\right)>0$ we may choose a neighborhood $U_{\delta}=\left\{s:\left|\operatorname{Re}(s)-\operatorname{Re}\left(s_{1}\right)\right|<\delta\right\}$ in the right half-plane so that $N-\operatorname{Re}\left(\frac{s}{2}\right) M \geq n+1$, for all $s \in U_{\delta}$. Therefore, for $s \in U_{\delta}$

$$
\left|h(x) m(x)^{\frac{s}{2}}\right| \leq C^{\prime}|| h \|_{N}(1+|x|)^{-(n+1)}
$$

and

$$
\left|T_{s}(h)\right| \leq C^{\prime}\|h\|_{N}\left(\int_{\mathbf{R}^{N}}(1+|x|)^{-(n+1)} d x\right)=C\|h\|_{N} .
$$

Therefore, $T_{s}$ is a tempered distribution for $\operatorname{Re}(s)>0$. Furthermore, there is a convergent power series expansion $T_{s}(h)=\sum_{m=0}^{\infty} T^{m}(h)\left(s-s_{1}\right)^{m}$. We see that each $T^{m}$ is a tempered distribution as follows. Let $h_{j} \rightarrow h$ in $\mathcal{S}\left(\mathbf{R}^{n}\right)$. Then by (B.4) $T_{s}\left(h_{j}\right) \rightarrow T_{s}(h)$ uniformly on compact sets (in the right half-plane). Therefore, $T^{m}\left(h_{j}\right) \rightarrow T^{m}(h)$ (as the coefficients in the expansion are derivatives of $T_{s}(h)$ ).

Now consider an arbitrary $s_{1} \in$ C. Choose $k$ in (B.1) large enough so that $\operatorname{Re}\left(s_{1}\right)+k>0$. Write $b(s+2) \cdots b(s+2 k)=b_{1}(s)\left(s-s_{1}\right)^{d}$ with $b_{1}\left(s_{1}\right) \neq 0$ and $d$ a nonnegative integer. Then

$$
T_{s}(h)=\frac{1}{b_{1}(s)} \frac{1}{\left(s-s_{1}\right)^{d}} T_{s+k}\left(m\left(\partial_{x}\right) h(x)\right) .
$$

Expanding $T_{s+k}$ about $s_{1}+k$ (as above) gives

$$
\begin{aligned}
T_{s}(h) & =\frac{1}{b_{1}(s)} \frac{1}{\left(s-s_{1}\right)^{d}} \sum_{m=0}^{\infty} T^{m}\left(m\left(\partial_{x}\right) h(x)\right)\left(s-s_{1}\right)^{m} \\
& =\frac{1}{b_{1}(s)} \sum_{m=-d}^{\infty} T^{m+d}\left(m\left(\partial_{x}\right) h(x)\right)\left(s-s_{1}\right)^{m} .
\end{aligned}
$$

As each $T^{m}$ in (B.5) is tempered the Lemma is proved.

Now suppose $g(s)$ is an entire function and set $\tilde{T}_{s}(h)=g(s) T_{s}(h)$ for each $h \in \mathcal{S}(\mathfrak{n})$. By the above Lemma $\tilde{T}_{s}$ is a meromorphic family of tempered distributions and we may write

$$
\tilde{T}_{s}(h)=g_{1}(s) \sum_{m \geq-d_{1}} T^{m}(h)\left(s-s_{1}\right)^{m}
$$

where $g(s)=g_{1}(s)\left(s-s_{1}\right)^{d_{1}}, g_{1}\left(s_{1}\right) \neq 0$.

Corollary B.7. Suppose $s_{1}$ is a possible pole of $\tilde{T}_{s}(h)$, for some $h \in \mathcal{S}\left(\mathbf{R}^{n}\right)$. If $\tilde{T}_{s_{1}}(h)<\infty$ for all $h \in C_{0}^{\infty}\left(\mathbf{R}^{n}\right)$ (=compactly supported functions) then $\tilde{T}_{s_{1}}(h)$ is finite for all $h \in \mathcal{S}\left(\mathbf{R}^{n}\right)$ and defines a tempered distribution.

Proof. If $\tilde{T}_{s_{1}}(h)<\infty$ for all $h \in C_{0}^{\infty}\left(\mathbf{R}^{n}\right)$ then $T^{m}(h)=0$ for all $m=-1,-2, \ldots,-d_{1}$ and $h \in C_{0}^{\infty}\left(\mathbf{R}^{n}\right)$. As each $T^{m}$ is tempered, $T^{m}(h)=0$ for all $m=-1,-2, \ldots,-d_{1}$ and $h \in \mathcal{S}\left(\mathbf{R}^{n}\right)$. Now

$$
T_{s_{1}}(h)=g_{1}\left(s_{1}\right) T^{0}(h),
$$

a tempered distribution.

\section{ApPendix C. RANK ONE CASE}

Many of the arguments given in this article use induction on the rank of $\mathfrak{n}$. In this appendix we collect the facts about the rank one case which are used. First we make some observations about the Lie algebras $\mathfrak{g}_{1}$ and $\mathfrak{n}_{1}$, when $\mathfrak{g}$ is on Table 1 . 
Recall that $\mathfrak{g}_{1}$ is the Lie algebra generated by the root spaces for $\pm 2 \epsilon_{1}$. This is a simple Lie algebra and, although $\mathfrak{g}_{1}$ does not in general satisfy 2.1, the integer $e+1$ (the dimension of the root space for the long root) is the same as for $\mathfrak{g}$. From Table 1 we see that $\mathfrak{g}_{1}$ is

$$
\begin{aligned}
& \mathfrak{g l}(2, \mathbf{R}) \text { in cases } 1-4, \\
& \mathfrak{s l}(2, \mathbf{C}) \text { in cases } 5-9, \\
& \mathfrak{s p}(1,1) \text { in case } 10, \\
& \mathfrak{s l}(2, \mathbf{H}) \text { in case } 11 \text { and } \\
& \mathfrak{s o}(p, 1) \text { in case } 12 .
\end{aligned}
$$

Note that $\operatorname{dim}\left(\mathfrak{n}_{1}\right)=e+1$. The following lemma computes $\nabla(X)$ and $\bar{\nabla}(Y)$ for each $\mathfrak{g}_{1}$. We express $\nabla$ and $\bar{\nabla}$ in terms of $\langle\rangle=,-\frac{1}{4 m} B_{\mathfrak{g}}$.

Lemma C.1. Suppose that $\mathfrak{n}$ has rank 1 . Then $\nabla(X)=\|X\|$ and $\bar{\nabla}(Y)=\|Y\|$.

Proof. It is enough to compute just $\bar{\nabla}(Y)=\|Y\|$ since for $X=\theta(Y),\|X\|^{2}=\langle\theta(X), X\rangle=\langle Y, \theta(Y)\rangle=$ $\|Y\|^{2}$. We do a case-by-case calculation. The cases of $\mathfrak{s l}(2, \mathbf{R})$ and $\mathfrak{s l}(2, \mathbf{C})$ may be done simultaneously. Here

and $\mathfrak{n}=\theta(\overline{\mathfrak{n}})$. Also

$$
\overline{\mathfrak{n}}=\left\{Y=\left(\begin{array}{ll}
0 & 0 \\
y & 0
\end{array}\right)\right\} \text { with } y \in \mathbf{R} \text { or } \mathbf{C}
$$

$$
\mathfrak{a}=\left\{\left(\begin{array}{cc}
a & 0 \\
0 & -a
\end{array}\right): a \in \mathbf{R}\right\}
$$

and the Weyl group element is represented by

$$
w=\left(\begin{array}{cc}
0 & 1 \\
-1 & 0
\end{array}\right)
$$

We need to compute $\bar{\nabla}(Y)=\mathrm{e}^{\epsilon_{1}\left(a\left(w \bar{n}_{Y}\right)\right)}$. Consider the standard representation on $\mathbf{C}^{2}$ with the usual hermitian metric $($,$) . Then$

$$
\begin{aligned}
\mathrm{e}^{\epsilon_{1}\left(a\left(w \bar{n}_{Y}\right)\right)} & =\left|\left(\left(\begin{array}{l}
1 \\
0
\end{array}\right), w \bar{n}_{Y} \cdot\left(\begin{array}{l}
1 \\
0
\end{array}\right)\right)\right| \\
& =\left|\left(\left(\begin{array}{l}
1 \\
0
\end{array}\right),\left(\begin{array}{cc}
y & 1 \\
-1 & 0
\end{array}\right)\left(\begin{array}{l}
1 \\
0
\end{array}\right)\right)\right| \\
& =|y| .
\end{aligned}
$$

Now we need to compare $|y|$ with $\|Y\|$. Since $\frac{1}{4 m} B(\xi, \eta)=\operatorname{Re}\left(\operatorname{Tr}(\xi \eta)\right.$ we have $\|Y\|^{2}=\operatorname{Tr}\left(\bar{Y}^{t} Y\right)=|y|^{2}$. Therefore, $\bar{\nabla}(Y)=\|Y\|$. The other cases are done similarly.

For the cases when $\mathfrak{n}$ is of rank one, the functional equation is simply a statement about the distribution $|x|^{-1}$ in euclidean space $\mathbf{R}^{m}$ and is well-known. A good reference is [8, vol. 1, Ch. II.3].

Proposition C.2. Consider $\mathbf{R}^{m}$ for any integer $m$ and $f \in \mathcal{S}\left(\mathbf{R}^{m}\right)$. With Fourier transform defined by

$$
\widehat{f}(y)=\int_{\mathbf{R}^{m}} f(x) e^{-2 \pi i\langle y, x\rangle} d x,
$$

the functional equation takes the form

$$
\frac{\pi^{\frac{m-s}{2}}}{\Gamma\left(\frac{m-s}{2}\right)} \int_{\mathbf{R}^{m}} f(x)|x|^{-s} d x=\frac{\pi^{\frac{s}{2}}}{\Gamma\left(\frac{s}{2}\right)} \int_{\mathbf{R}^{m}} \widehat{f}(y)|y|^{s-m} d y .
$$

The following Proposition is also well-known, see for example [8, vol 4, Ch. II.3.6]. We give a short proof here since our proof of Theorem 6.4 refers to formula (C.5) occurring below. 
Proposition C.4. The distributions $R_{s}^{1}(f)=\frac{\pi^{\frac{-s+m}{2}}}{\Gamma\left(\frac{s}{2}\right)}|x|^{-s}$ are positive if and only if $s \leq m$.

Proof. When $s<m$ the function $|x|^{-s}$ is a locally $L_{1}$ positive function (and $\Gamma\left(\frac{-s+m}{2}\right)>0$ ) so the distribution is clearly positive. For $s=m, R_{m}^{1}=\frac{\frac{m}{2}}{\Gamma\left(\frac{m}{2}\right)} \cdot \delta_{0}$, a positive distribution.

To see that $R_{s}^{1}$ is not positive for $s \in \mathbf{C} \backslash(-\infty, m]$ consider the positive Schwartz functions

$$
\varphi_{1}(x)=|x|^{2} \mathrm{e}^{-|x|^{2}} \text { and } \varphi_{2}(x)=\mathrm{e}^{-|x|^{2}} .
$$

We claim that

$$
\begin{aligned}
& R_{s}^{1}\left(\varphi_{1}\right)=\frac{1}{4} \pi^{\frac{-s+m}{2}} \operatorname{Vol}\left(S^{m-1}\right) \cdot(-s+m) \\
& R_{s}^{1}\left(\varphi_{2}\right)=\frac{1}{2} \pi^{\frac{-s+m}{2}} \operatorname{Vol}\left(S^{m-1}\right) .
\end{aligned}
$$

Then

$$
\frac{R_{s}^{1}\left(\varphi_{1}\right)}{R_{s}^{1}\left(\varphi_{2}\right)}=\frac{-s+m}{2}
$$

so both $R_{s}^{1}\left(\varphi_{i}\right), i=1,2$ cannot be positive for $s \in \mathbf{C} \backslash(-\infty, m)$.

By analytic continuation it suffices to check the claim for $s \ll 0$.

$$
\begin{aligned}
R_{s}^{1}\left(\varphi_{1}\right) & =\frac{\pi^{\frac{-s+m}{2}}}{\Gamma\left(\frac{-s+m}{2}\right)} \int_{\mathbf{R}^{m}} \mathrm{e}^{-|x|^{2}}|x|^{-s+2} d x \\
& =\frac{\pi^{\frac{-s+m}{2}}}{\Gamma\left(\frac{-s+m}{2}\right)} \int_{S^{m-1}} \int_{0}^{\infty} \mathrm{e}^{-r^{2}} r^{-s+m+1} d r d \sigma \\
& =\frac{\pi^{\frac{-s+m}{2}}}{\Gamma\left(\frac{-s+m}{2}\right)} \operatorname{Vol}\left(S^{m-1}\right) \int_{0}^{\infty} \mathrm{e}^{-t} t^{\frac{-s+m+1}{2}} \frac{d t}{2 \sqrt{t}} d t \\
& =\frac{1}{2} \pi^{\frac{-s+m}{2}} \operatorname{Vol}\left(S^{m-1}\right) \frac{\Gamma\left(\frac{-s+m}{2}+1\right)}{\Gamma\left(\frac{-s+m}{2}\right)}=\frac{1}{4} \pi^{\frac{-s+m}{2}} \operatorname{Vol}\left(S^{m-1}\right) \cdot(-s+m) .
\end{aligned}
$$

The second part of the claim has a similar proof.

\section{REFERENCES}

[1] L. Barchini and M. Sepanski, Application of restriction of Fourier transforms to an example from representation theory, Pacific J. Math. 198 (2001), no. 2, 265-293.

[2] I.N. Bernstein and S.I. Gelfand, Meromorphic properties of the $P^{\lambda}$, Funct. Anal. App., 3 (1969), 68-69.

[3] N. Bopp and H. Rubenthaler, Fonction zêta associée à la série principale sphérique de certains espaces symétriques, Ann. Sci. École Norm. Sup. (4) 26 (1993), no. 6, 701-745.

[4] N. Bopp and H. Rubenthaler, Une fonction zêta associée à certaines familles d'espaces symtriques rèels, C. R. Acad. Sci. Paris Sr. I Math. 325 (1997), no. 4, 355-360.

[5] A. Dvorsky and S. Sahi, Explicit Hilbert spaces for certain unipotent representations, II, Invent. Math. 138 (1999), no. 1, 203-224.

[6] A. Dvorsky and S. Sahi, Explicit Hilbert spaces for certain unipotent representations, III, preprint.

[7] J. Faraut and A. Koranyi, Analysis on Symmetric Cones, Oxford University Press, Oxford, 1994.

[8] I.M. Gelfand and G.E. Shilov, Generalized function, Volumes 1 and 4. Academic press, New York, 1964.

[9] S. Gindikin, Invariant generalized functions in homogeneous domains, Journal of Functional Analysis and Applications $\mathbf{9}$, (1975), no. 1, 56-58.

[10] A. W. Knapp, Representation theory of semisimple Lie groups. An overview based on examples. Princeton University Press, Princeton, New Jersey, 1986.

[11] S. Kaneyuki, The Sylvester's law of inertia in simple graded Lie algebras, J. Math. Soc. Japan 50, (1998), no. 3, 593-614.

[12] T. Kobayashi and B. Ørsted, Analysis of the minimal representation of $O(p, q)$, III, preprint.

[13] B. Kostant and S. Sahi, Jordan algebras and Capelli identities, Invent. Math. 112, (1993), 647-664. 
[14] L. H. Loomis, An introduction to abstract harmonic analysis. Van Nostrand Co., Princeton, NJ, 1953.

[15] I. Muller, Décomposition orbitale des espaces préhomognes réguliers de type parabolique commutatif et application, C. R. Acad. Sci. Paris Sr. I Math. 303 (1986), no. 11, 495-498.

[16] I. Muller, Structure and orbits of certain prehomogeneous vector spaces related with orthogonal roots, Proc. Japan Acad. Ser. A Math. Sci. 72, no. 5, (1996), 104-107.

[17] I. Muller, On local zeta functions associated to prehomogeneous vector spaces of commutative parabolic type. Part I: local coefficients, preprint.

[18] S. Rallis and G. Schiffmann, Distributions invariantes par le groupee orthogonal in Analyse Harmonique sur las Groupes de Lie (P. Eymard, J. Faraut, G. Schiffmann and R.Takahashi, eds.), Lecture Notes in Mathematics vol. 497 Springer-Verlag, New York, 1975

[19] H. Rossi and M. Vergne, Analytic continuation of the holomorphic discrete series of a semi-simple Lie group, Acta Math. 136 (1976), no. 1-2, 1-59.

[20] S. Sahi, Jordan algebras and degenerate principal series, J. Reine Angew. Math. 462, (1995) 1-18.

[21] M. Sato, Theory of prehomogeneous vector spaces, Sugakuno Ayumi 15 (1963), 85-157.

[22] M. Sato and T. Shintani, On zeta functions associated with prehomogeneous vector spaces, Ann. of Math. (2) 100, (1974) 131-170.

[23] H. Schlichtkrull, Hyperfunctions and harmonic analysis on symmetric spaces. Birkhauser, Boston, 1984.

[24] G. Shimura, Generalized Bessel functions on symmetric spaces, J. Reine Angew. Math. 509 (1999), 35-66.

[25] T. Shintani, On the Dirichlet series whose coefficients are class numbers of integral cubic forms, J. Math. Soc. Japan 24, (1972), 132-188.

[26] D. A. Vogan and N. Wallach, Intertwining operators for real reductive groups, Adv. Math. 82, (1990), no. 2, $203-243$.

[27] A. Weil, Sur certains groupes d'opérateurs unitaires, Acta Math. 111, (1964) 143-211

Oklahoma State University, Mathematics Department, Stillwater, Oklahoma 74078

E-mail address: leticia@math.okstate.edu

Baylor University, Mathematics Department, Waco, Texas 76798

E-mail address: Mark_Sepanski@baylor.edu

Oklahoma State University, Mathematics Department, Stillwater, Oklahoma 74078

E-mail address: zierau@math.okstate.edu 